Provided for non-commercial research and education use. Not for reproduction, distribution or commercial use.

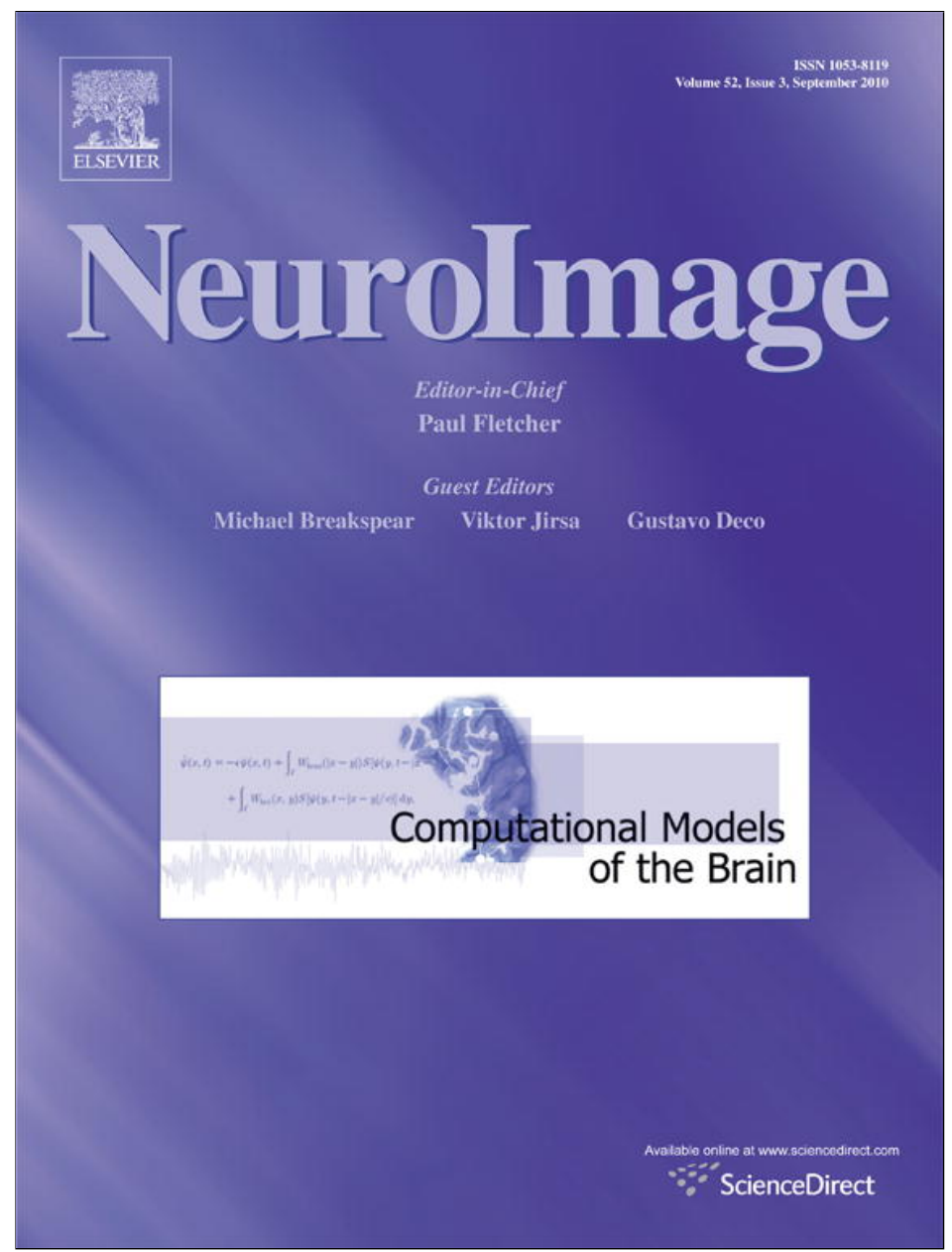

This article appeared in a journal published by Elsevier. The attached copy is furnished to the author for internal non-commercial research and education use, including for instruction at the authors institution and sharing with colleagues.

Other uses, including reproduction and distribution, or selling or licensing copies, or posting to personal, institutional or third party websites are prohibited.

In most cases authors are permitted to post their version of the article (e.g. in Word or Tex form) to their personal website or institutional repository. Authors requiring further information regarding Elsevier's archiving and manuscript policies are encouraged to visit:

http://www.elsevier.com/copyright 


\title{
Understanding the relationships between spike rate and delta/gamma frequency bands of LFPs and EEGs using a local cortical network model
}

\author{
Alberto Mazzoni ${ }^{\text {a,b }}$, Kevin Whittingstall ${ }^{\text {c }}$, Nicolas Brunel ${ }^{\text {b,d,e }}$, Nikos K Logothetis ${ }^{\text {c,f }}$, Stefano Panzeri ${ }^{\text {a,* }}$ \\ a Department of Robotics, Brain and Cognitive Sciences, Italian Institute of Technology, via Morego 30, 16163 Genoa, Italy \\ ${ }^{\mathrm{b}}$ Institute for Scientific Interchange, viale Settimio Severo 65, 10133, Turin, Italy \\ c Max Planck Institute for Biological Cybernetics, Spemannstrasse 38, 72076, Tuebingen, Germany \\ d Laboratory of Neurophysics and Physiology, Université Paris Descartes, Rue des Saints Pères 45, Paris, France \\ e CNRS UMR 8119, Paris, France \\ ${ }^{\mathrm{f}}$ Division of Imaging Science and Biomedical Engineering, University of Manchester, The Mill, PO Box 88, Manchester M60 1QD, UK
}

\section{A R T I C L E I N F O}

\section{Article history:}

Received 10 September 2009

Revised 4 December 2009

Accepted 8 December 2009

Available online 21 December 2009

\section{Keywords:}

Electroencephalography

Delta rhythm

Gamma rhythm

Integrate-and-fire network

Field potential

Natural stimuli

Oscillations

Cross-frequency coupling

\begin{abstract}
A B S T R A C T
Despite the widespread use of EEGs to measure the large-scale dynamics of the human brain, little is known on how the dynamics of EEGs relates to that of the underlying spike rates of cortical neurons. However, progress was made by recent neurophysiological experiments reporting that EEG delta-band phase and gamma-band amplitude reliably predict some complementary aspects of the time course of spikes of visual cortical neurons. To elucidate the mechanisms behind these findings, here we hypothesize that the EEG delta phase reflects shifts of local cortical excitability arising from slow fluctuations in the network input due to entrainment to sensory stimuli or to fluctuations in ongoing activity, and that the resulting local excitability fluctuations modulate both the spike rate and the engagement of excitatory-inhibitory loops producing gamma-band oscillations. We quantitatively tested these hypotheses by simulating a recurrent network of excitatory and inhibitory neurons stimulated with dynamic inputs presenting temporal regularities similar to that of thalamic responses during naturalistic visual stimulation and during spontaneous activity. The network model reproduced in detail the experimental relationships between spike rate and EEGs, and suggested that the complementariness of the prediction of spike rates obtained from EEG delta phase or gamma amplitude arises from nonlinearities in the engagement of excitatory-inhibitory loops and from temporal modulations in the amplitude of the network input, which respectively limit the predictability of spike rates from gamma amplitude or delta phase alone. The model suggested also ways to improve and extend current algorithms for online prediction of spike rates from EEGs.
\end{abstract}

(C) 2009 Elsevier Inc. All rights reserved.

\section{Introduction}

Electroencephalography (EEG) is one the most important tools for non-invasively studying brain activity in humans at fine time resolution (Lopes da Silva and Van Rotterdam, 1987; Nunez, 1981). Despite its wide use in clinical applications and in neurophysiological research, the exact relationships between the surface EEG and the underlying physiological events at the cellular and network level remain only partly known. Early studies (Creutzfeldt et al., 1966a, b; Klee et al., 1965) demonstrated that a prominent contribution to cortical surface EEGs comes from excitatory and inhibitory synaptic potentials (mostly from pyramidal neurons but perhaps also from spiny and aspiny stellate cells, see (Murakami and Okada, 2006)), and from afterdischarges not directly related to cellular activity (Creutzfeldt et al., 1966a, b; Klee et al., 1965). Other studies (Granit et al., 1963; Juergens et al., 1999; Kamondi et al., 1998; Mitzdorf,

\footnotetext{
* Corresponding author.

E-mail address: stefano.panzeri@iit.it (S. Panzeri).
}

1987) showed that these mechanisms also contribute to the generation of the Local Field Potential (LFP), an intracortical signal which shares similarities with the EEG but is more localized (Katzner et al., 2009). However, we still do not know which aspects of the time course and frequency content of the surface EEG allow an estimation of the time course of the spiking activity of cortical projection neurons, i.e. the output of the cortical site. This is clearly an important question to address for several reasons. First, progress in estimating the strength and timing of cortical spike rates from EEGs would greatly increase our understanding of the neural computations underlying the recorded EEG signal. Second, understanding how macroscopic and mesoscopic signals such as EEGs and LFPs relate to the output of a very local neuronal computation (whose results is carried by the spikes of pyramidal neurons) is a fundamental empirical step in constructing models linking large scale dynamics of cortex to computations of local networks.

Recently, we made progress in this direction (Whittingstall and Logothetis, 2009) by showing that, in macaque primary visual cortex, the time course of the spike rate can be predicted by a combination 
of the instantaneous delta-band $(2-4 \mathrm{~Hz})$ phase and gamma-band (30-100 Hz) amplitude of the concurrently recorded surface EEG. This was observed both during visual stimulation with naturalistic movies and during stimulus-free periods. Consistent findings were also obtained when predicting spike rates from intracortical LFPs rather than from EEGs (Rasch et al., 2008; Whittingstall and Logothetis, 2009). Interestingly, the cross-frequency coupling (i.e. the coupling of the phase of a slower frequency with the amplitude of a faster rhythm) which has been shown to determine the strength and timing of spiking activity in visual cortex (Whittingstall and Logothetis, 2009) has also been consistently observed in neocortex (Canolty et al., 2006; Lakatos et al., 2005) and hippocampus (Bragin et al., 1995; Lisman, 2005) and is thought to be central for a number of cognitive and sensory processes (Jensen and Colgin, 2007; Lisman, 2005; Lisman and Idiart, 1995; Schroeder and Lakatos, 2009).

These findings raise the important question of what are the mechanisms which generate cross frequency-coupling, and how cross-frequency coupling correlates to the timing and strength of spiking activity. Answering these questions would not only allow better insights into the mechanism regulating cortical dynamics, but has obvious implications for improving the prediction of spiking activity from EEGs and LFPs.

Here we aim at explaining these empirical findings by hypothesizing that the EEG-LFP delta phase reflects shifts of the cortical excitability arising from low-frequency (delta range) fluctuations in the strength of the input to the local network, and that these changes in excitability modulate both the output spike rate and the engagement of excitatory-inhibitory loops producing gamma-band oscillations, which in turn leads to the observed three-way relationships between spike rate, gamma amplitude and delta phase. Slow (delta range) input fluctuations may be either mediated by thalamocortical connections and arise from slow variations in thalamic firing reflecting responses to the relatively slow and regular changes present in naturalistic stimuli, or may be mediated by cortico-cortical connections and originate from slow and spatially extended fluctuations of ongoing cortical activity.

To test quantitatively this hypothesis, we simulated a recurrent network of integrate-and-fire neurons with excitatory-inhibitory connections stimulated with dynamic inputs with temporal regularities similar to that of thalamic responses during naturalistic visual stimulation and during spontaneous activity; we then carefully studied the dependence between the simulated EEG-LFP frequency bands and the spike rate of the simulated pyramidal neurons and how this dependence is modulated by different biophysical mechanisms; and we compared in detail the spike-EEG/LFP relationships found in the model and in real EEG/LFP recordings of awake and anaesthetized macaques during stimulation with naturalistic movies or in absence of visual stimuli.

\section{Methods}

All experiments conducted on macaques were approved by the local authorities (Regierungspräsidium Tübingen) and are in full compliance with the guidelines of the European Community (EUVD $86 / 609 /$ EEC) for the care and use of laboratory animal.

\section{Recordings of EEGs, LFPs and spike from the primary visual cortex of} awake macaques

Methods were fully reported elsewhere (Whittingstall and Logothetis, 2009), to which we refer for more details. In brief, EEG recordings were made from two non-anesthetized monkeys (Macaca mulatta) using a $\mathrm{Ag} / \mathrm{AgCl}$ ring electrode (impedance below $20 \mathrm{k} \Omega$ ) positioned over the visual cortex. EEG signals were amplified and filtered into a band of $0.2-250 \mathrm{~Hz}$ (Brain Products, Munich, Germany) and digitized at $5 \mathrm{kHz}$. The EEG ring electrode of interest was placed at the base of a recording chamber positioned over primary visual cortex
(V1), made from PEEK (polyetheretherketone; TecaPEEK, Nufringen, Germany) and secured to the skull. The EEG ring electrode rested on the skull and small circular openings (under the center of the EEG ring electrode) in the skull were made to access cortical neurons. In one monkey, a $5 \mathrm{~mm}$ circular patch was resected, while in the other monkey, a $2 \mathrm{~mm}$ circular patch was resected in order to access the underlying cortex. Tungsten microelectrodes (FHC Inc., Bowdoinham, Maine, 0.5 to $2 \mathrm{M} \Omega$ ) were lowered through the middle of the EEG ring electrode into the cortex in order to obtain spiking activity and LFPs. The extracellular signals obtained from these intracortical tungsten microelectrodes (whose tips were typically, but not always, positioned in the upper or middle cortical layers) were high-pass filtered $(1 \mathrm{~Hz}$, digital two pole Butterworth filter), amplified (Alpha Omega Engineering) and digitized at $20.83 \mathrm{kHz}$. A frontal EEG electrode placed on the scalp was used as reference.

The LFPs were extracted by band-pass filtering the neural signal in the 2- to $125-\mathrm{Hz}$ range and resampling at a rate of $250 \mathrm{~Hz}$. Filtering was done using Kaiser filters with sharp transition bandwidth $(1 \mathrm{~Hz})$, small passband ripple $(0.01 \mathrm{db})$ and high stopband attenuation $(60 \mathrm{~dB})$. A mirroring technique was used to reduce edge artifacts during filtering and forwards and backwards filtering was used to eliminate phase shifts. The very same filtering technique was applied to further bandpass all the real neural data and the simulated to create the signal bandpassed in the various frequency bands (such as delta and gamma band). The instantaneous amplitude and phase of the bandpassed signals were obtained by taking respectively the modulus and angle of the complex time series obtained through the Hilbert transform of the bandpassed signal. Circular statistics analysis on the so obtained phase distributions was performed with the CircStat MATLAB (The Mathworks, Natick, MA) Toolbox described in Berens (2009).

To extract spike times, following Quiroga et al. (2004), we bandpass filtered (with the same filtering techniques described above) the extracellular signal from intracortical electrode at $300-4000 \mathrm{~Hz}$, and we used for spike detection an amplitude threshold of 4 standard deviations (sds) of the mean amplitude. A spike was recognized as such only if the last spike occurred more than $1.5 \mathrm{~ms}$ earlier. This threshold approach for spike detection is appropriate for spike times but not for the isolation of single units. Thus, the spikes used for the analysis represented the spiking activity of a small population of cells rather than well-separated spikes from single neurons (Quiroga et al., 2004).

Visual stimuli consisted of naturalistic commercially available movies ( $30 \mathrm{~Hz}$ frame rate), from which 5 -s clips were presented on a computer screen (field of view: $90 \mathrm{~Hz}$ refresh); each stimulus was repeated 30-40 times per experimental session. Data were acquired while the animals performed a visual fixation task ( $9 \mathrm{~s}$ fixation period, 2.0-2.8 deg fixation window). Each 9-s-long trial consisted of $2 \mathrm{~s}$ of fixation of a small (0.2 deg) fixation spot on dark background, followed by $5 \mathrm{~s}$ of movie presentation during fixation of the same spot, finally followed by 2 of continued fixation of the spot on dark background. When analyzing movie-driven activity, we discarded from further analysis the first second of data as it mainly consisted of a transient response to the stimulus onset. We also analyzed data recorded from the awake monkey during the first period of fixation of spot on dark background prior to the movie, and we will refer for brevity to these data as collected during "spontaneous activity" to mean that they are collected in absence of the movie stimulus.

Recordings in primary visual cortex and in the lateral geniculate nucleus of anaesthetized monkeys

We also analyzed recordings from 76 sites in $\mathrm{V} 1$ and from 8 sites in the dorsal lateral geniculate nucleus (LGN) that were obtained in a separate set of experiments involving four adult rhesus monkeys (Macaca mulatta). Full details of experimental procedures were given elsewhere (Belitski et al., 2008; Rasch et al., 2008). In brief, recordings were obtained while the animals were anaesthetized (remifentanyl, 
$1 \mu \mathrm{g} / \mathrm{kg} / \mathrm{min}$ ), muscle-relaxed (mivacurium, $5 \mathrm{mg} / \mathrm{kg} / \mathrm{h}$ ) and ventilated (end-tidal $\mathrm{CO}_{2} 33 \mathrm{~mm} \mathrm{Hg}$, oxygen saturation $>95 \%$ ). Body temperature was kept constant and lactated Ringer's solution supplied $(10 \mathrm{ml} / \mathrm{kg} / \mathrm{h}$ ). Vital signs (SpO2, ECG, blood pressure, endtidal $\mathrm{CO}_{2}$ ) were continuously monitored. $1 \%$ ophthalmic solution (cyclopentolate hydrochloride) was applied to induce mydriasis and refractive errors were corrected using contact lenses. Microelectrodes (FHC Inc., Bowdoinham, Maine, 300-800 k $\Omega$ ) were positioned using a microdrive array system (Thomas Recording, Giessen, Germany). When recording from cortex, microelectrodes were arranged in a $4 \times 4$ square matrix (interelectrode spacing varied from $1 \mathrm{~mm}$ to $2.5 \mathrm{~mm}$ ). In some sessions, an additional array of two or four electrodes was additionally positioned in the dorsal LGN using an additional set of drives in order to record thalamic activity simultaneously to the recording of cortical activity. Signals were high-pass filtered $(1 \mathrm{~Hz}$, digital two pole Butterworth filter) and amplified using an Alpha Omega amplifier system (Alpha Omega Engineering) and digitized at $20.83 \mathrm{kHz}$. Binocular visual stimuli were presented at a resolution of 640x480 pixels (field of view: 30x23 degrees, 24 bit true color, $60 \mathrm{~Hz}$ refresh) using a fiber-optic system (Avotec, Silent Vision, Florida). Stimuli consisted of "naturalistic" complex and commercially available movies ( $30 \mathrm{~Hz}$ frame rate), from which 3.5- to 6-min long sequences were presented and repeated 30-40 times. In addition to recordings in response movies, for each recording session and site we also recorded 5-10 trials of 5-min long "spontaneous activity" when the input to the fiber-optic stimulus presentation system was blank.

LFP bands were extracted from the extracellular signal exactly as described above for recordings with awake monkeys. To extract spike times, the extracellular signal was filtered in the range of $500-3500 \mathrm{~Hz}$. The threshold for spike detection was set at 3.5 sds. A spike was recognized as such only if the last spike occurred $1 \mathrm{~ms}$ earlier. As for the awake macaque data, this simple method does not permit the isolation of single units.

\section{Simulations}

The model is similar to the one described in Brunel and Wang (2003) and then extended in Mazzoni et al. (2008) to include timevarying inputs. In brief, the main difference with respect to the model of Mazzoni et al. (2008) is that the thalamic input stimulus was modified to make it more realistic, and that a "slow noise" term was inserted in the corticocortical synapses (to simulate ongoing cortical activity fluctuations) rather than in the thalamocortical ones. Full details are given in the following, and a model description according to the scheme suggested by Nordlie et al. (2009) is reported in Table 1.

\section{Single neuron model and network structure}

The network is composed of 5000 neurons. 4000 excitatory neurons have synapses with AMPA-like characteristics to model pyramidal neurons, while 1000 have synapses with GABA-like characteristics to model interneurons. The network is randomly connected: the connection probability between any directed pair of cells is 0.2 (Holmgren et al., 2003; Sjostrom et al., 2001). Both pyramidal neurons and interneurons are described by leaky integrate and fire (LIF) dynamics with fixed threshold, fixed refractory time. The subthreshold dynamics of the membrane potential $V_{\mathrm{k}}(t)$ of each neuron $k$ was described by the following equation:

$\tau_{m} V_{k}^{\dot{k}}(t)=-V_{k}(t)+V_{A k}(t)-V_{G k}(t)$

where dots represent time derivatives $\tau_{\mathrm{m}}$ is the membrane time constant (20 ms for pyramidal neurons and $10 \mathrm{~ms}$ for interneurons) and $V_{\mathrm{Ak}}$ and $V_{\mathrm{Gk}}$ are respectively the absolute values of the AMPAtype and GABA-type post-synaptic currents (in membrane potential units for convenience) on neuron $k$. Potentials are measured such in a way that the resting potential is set at 0 .
Table 1

Model summary.

\begin{tabular}{|c|c|}
\hline \multicolumn{2}{|l|}{ Model summary } \\
\hline Populations & $\begin{array}{l}\text { Four: excitatory (4000), inhibitory (1000), thalamic input, } \\
\text { non-local cortical input. }\end{array}$ \\
\hline Topology & - \\
\hline Connectivity & $\begin{array}{l}\text { Random directed pair connections with probability } 0.2 \text {. } \\
\text { Delay: } 1 \text { ms. Different synaptic weights for each kind of } \\
\text { connection (see Table } 2 \text { ). }\end{array}$ \\
\hline Neuron model & $\begin{array}{l}\text { Leaky integrate and fire, fixed voltage threshold ( } 18 \mathrm{mV} \\
\text { above resting potential), fixed reset potential ( } 11 \mathrm{mV} \text { above } \\
\text { resting potential), and fixed absolute refractory time ( } 2 \mathrm{~ms} \\
\text { for excitatory neurons, } 1 \mathrm{~ms} \text { for inhibitory neurons). }\end{array}$ \\
\hline Channel models & - \\
\hline Synapse model & $\begin{array}{l}\text { Difference of exponentials (AMPA and GABA), with different } \\
\text { rise and decay time for each kind of connection (see Table 2). }\end{array}$ \\
\hline Plasticity & - \\
\hline Inputs & $\begin{array}{l}\text { Thalamic input: Poisson spike trains to all neurons with time } \\
\text { varying rate based on LGN spiking activity recorded in } \\
\text { anesthetized monkey V1 during move presentation (movie } \\
\text { condition) or during blank screen presentation } \\
\text { (spontaneous condition). Non local cortical input: Poisson } \\
\text { spike trains to all neurons with time varying rate given by } \\
\text { Ornstein Uhlenbeck process with cut-off frequency of } 10 \mathrm{~Hz} \text {. }\end{array}$ \\
\hline Measurements & $\begin{array}{l}\text { Spiking activity and EEG-LFP, the latter given by sum of } \\
\text { absolute values of AMPA and GABA currents. }\end{array}$ \\
\hline
\end{tabular}

The neuron $k$ fires if the potential $V_{\mathrm{k}}$ crosses the threshold $V_{\mathrm{thr}}$ (18 $\mathrm{mV}$ above resting potential). When a neuron fires, all the postsynaptic neurons are affected by the spike after a latency of $1 \mathrm{~ms}$ (according to Eqs. (1.2) and (1.3)), and the potential of neuron $k$ is reset and kept at a value $V_{\mathrm{r}}(11 \mathrm{mV})$ during a refractory period $\tau_{\text {ref }, k}$ ( $1 \mathrm{~ms}$ if neuron $k$ belongs to interneurons, $2 \mathrm{~ms}$ if it belongs to pyramidal neurons).

Synaptic currents are the linear sum of contributions induced by single pre-synaptic spikes, which are described by a difference of exponentials. The AMPA and GABA compound post-synaptic currents (PSC) $V_{\mathrm{Ak}}$ and $V_{\mathrm{Gk}}$ of neuron $k$ are determined by the spikes emitted by the pre-synaptic neurons of the network and by the external inputs (modelling both inputs from the neighbouring areas of the cortex and inputs from the thalamus). The values of the PSCs are obtained using auxiliary variables $X_{\mathrm{A} / \mathrm{G}, k}$, using the following equations

$$
\begin{aligned}
& \tau_{d A} V_{A k}^{\dot{A}}(t)=-V_{A k}(t)+X_{A k} \\
& \tau_{r A} X_{A k}^{*}(t)=-X_{A k}(t)+\tau_{m}\left(J_{k, p y r}\left(\sum_{n \in p y r} C_{k, n} \delta\left(t-t_{n}^{*}-\tau_{L}\right)+S_{k}^{C}(t)\right)\right. \\
& +J_{k, \text { thal }} S_{k}^{T h}(t) \\
& \tau_{d G} V_{G k}^{\cdot}(t)=-V_{G k}(t)+X_{G k} \\
& \tau_{r G} X_{G k}^{\cdot}(t)=-X_{G k}(t)+\tau_{m}\left(J_{k, \text { inter }}\left(\sum_{n \in \text { inter }} C_{k, n} \delta\left(t-t_{n}^{*}-\tau_{L}\right)\right)\right)
\end{aligned}
$$

where $t_{\mathrm{n}}^{*}$ are the times at which the neuron $\mathrm{n}$ fired and $\mathrm{C}$ is the connectivity matrix, in which $C_{k, n}$ is 1 if $\mathrm{n}$ projects to $k$ and 0 otherwise. $\tau_{\mathrm{dA} / \mathrm{G}}$ and $\tau_{\mathrm{rA} / \mathrm{G}}$ are respectively the decay and rising times of the AMPA and GABA synapses, $\tau_{\mathrm{L}}$ is the latency of post-synaptic currents ( $1 \mathrm{~ms}), J_{\mathrm{k}, \mathrm{pyr} / \mathrm{thal} / \mathrm{int}}$ is the synaptic strength of cortical AMPA synapses/ thalamic AMPA synapses/GABA synapses on the population of neurons to which $k$ belongs (interneurons or pyramidal neurons). $S_{k}^{C / \text { Thal }}(t)$ is the number of excitatory synapses of neuron $k$ activated by stimuli from the neighbouring areas of the cortex / from the thalamus (see Inputs subsection). The values of synaptic parameters are shown in Table 2. All values are of the order of magnitude of the values measured experimentally (Buhl et al., 1997. 
Table 2

Synaptic parameters.

\begin{tabular}{|c|c|c|c|c|c|c|}
\hline \multirow[t]{2}{*}{ Target } & \multicolumn{2}{|l|}{ GABA } & \multicolumn{2}{|c|}{ AMPA (cortex) } & \multicolumn{2}{|c|}{ AMPA (thalamus) } \\
\hline & Pyramidals & Interneurons & Pyramidals & Interneurons & Pyramidals & Interneurons \\
\hline Rise time (ms) & 0.25 & 0.25 & 0.4 & 0.2 & 0.4 & 0.2 \\
\hline Decay time (ms) & 5 & 5 & 2 & 1 & 2 & 1 \\
\hline Strength (mV) & 1.7 & 2.7 & 0.42 & 0.7 & 0.55 & 1.1 \\
\hline
\end{tabular}

Gabernet et al., 2005; Gil and Amitai, 1996; Gupta et al., 2000; Tamas et al., 1998). Modifying these values affects quantitatively but not qualitatively the results as long as recurrent inhibition is stronger than recurrent excitation (see Section Robustness to parameter changes of the behaviour of the simulated network and also Brunel and Wang (2003) and Mazzoni et al. (2008).

\section{Inputs}

Each neuron $k$ receives two excitatory inputs from outside the network: $S_{k}^{\text {Thal }}(t)$ from the thalamus and $S_{k}^{\text {Cl }}(t)$ from the afferent areas of the cortex, conveyed by synapses of different strengths (see Synapse model section). These synapses are activated by random Poisson spike trains, with time-varying rates which are identical for all neurons. Input rate to corticocortical and thalamocortical synapses are generated as follows. The input rate to the thalamocortical synapses $v^{\text {Thal }}(t)$ was a simple time-independent or sinusoidal function of time when investigating "artificial" inputs with a simplified dynamics (Figs. 3 and 4), while when considering naturalistic stimulation dynamics was constructed from real LGN spike rates as follows. The naturalistic input was built from $1 \mathrm{~min}$ of extracellular LGN recordings of spike times from anesthetized macaque presented binocularly with natural movie scenes (movie condition) or with blank screen (spontaneous condition). The spike time sequence was smoothed with a 20-ms Gaussian window convolution. The resulting time series was normalized to set the average input per neuron in the movie input stimulation condition to $1.5 \mathrm{spikes} / \mathrm{ms} /$ cell. The latter is a realistic estimate of the total input to real pyramidal neurons, taking into account that they receive excitatory inputs from approximately $\sim 120$ thalamic neurons (Latawiec et al., 2000) each one firing 12 spikes/s (Dan et al., 1996; Lesica et al., 2007; Whittingstall and Logothetis, 2009) during stimulation with movies. The same normalization factor between measured spike rates and model input rates, determined as above from responses to movies, was used for spontaneous activity and naturalistic movie inputs. The input rate from the corticocortical synapses $v^{C}(t)$ represents non-specific ongoing contributions from different areas of cortex. Since cortical ongoing activity has the most power in the slow frequency range, and since the slow frequencies are the ones with higher spatial extension and thus are more likely to summate, these non-specific contributions were modelled to have the maximal power in a range below a cutoff frequency $\tau_{C}$ which was set to $10 \mathrm{~Hz}$ (and whose precise value had a negligible impact on the results as long as it remained below $20 \mathrm{~Hz}$ (Mazzoni et al. 2008)). The rate $v^{C}(t)$ activating the corticocortical synapses was generated according to an Orstein-Uhlenbeck process, as follows:

$\tau_{C} \dot{v}^{C}(t)=\mu_{C}-v^{C}(t)+\sigma_{C} \sqrt{2 \tau_{C}} \eta(t)$

where $\mu_{\mathrm{C}}$ is the average value of 1.5 spikes $/ \mathrm{ms} /$ cell, $\sigma_{\mathrm{C}}$ the standard deviation of 0.5 spikes/ms and $\eta(t)$ a Gaussian white noise process. The latter parameter values were chosen so that the input coefficient of variation across time matched the one of the spikes recorded in V1 during movie stimulation, and the LFP from the simulated network matched the information values of power of LFPs in the $1-150 \mathrm{~Hz}$ range obtained in V1 during naturalistic movie presentation (see (Mazzoni et al., 2008)). This ensured the simulation of ongoing activity matched the trial-to-trial variations in real data.

\section{Computation of simulated LFPs and EEGs}

Since a prominent contribution to real cortical LFP and EEG arises from current flows due to synaptic activity, we computed the LFP/EEG signal generated by the network simply as the sum of the absolute values of AMPA and GABA currents, as in Mazzoni et al. (2008). In computing the simulated LFP/EEG we summed only currents from synapses of pyramidal neurons, under the assumption that pyramidal neurons contribute more due to their approximate open field arrangement. The LFP/EEG signal was taken with a negative sign to be directly comparable with experimental recordings. Although more detailed quantifications of signals such as LFPs from simulated networks are in principle possible (see e.g.(Pettersen and Einevoll, 2008)), we note that the simple quantification we chose was enough to reproduce all the features of interest of experimentally recorded LFPs and EEGs (see Results), thereby suggesting that our simple description of LFPs and EEGs was sufficient for the present purpose. Moreover, we verified (Supplemental Fig. S1) that all main results in this study were robust to changes in the definition of LFP/EEG signal considering different weights to GABA currents (ranging from $0 \%$ to $50 \%$ more than AMPA currents) or adding a small contribution (up to $10 \%$ ) from the currents computed from synapses of interneurons (Murakami and Okada, 2006).

\section{Numerical implementation of model}

Model algorithms were developed in C. Equations were solved using a second-order Runge-Kutta scheme with a time step of $0.05 \mathrm{~ms}$.

\section{Results}

Summary of relationship between spike rate, gamma amplitude and delta phase in visual cortex

Before analyzing the model of the relationship between the time course of spiking activity and the frequency components of LFPs and EEGs, we present and analyze the basic experimental findings in visual cortex of awake and anaesthetized macaques. The core of these findings was reported by Whittingstall and Logothetis (2009) for the case of the awake monkey. Here we summarize their results by analyzing them in exactly the same way that will be later applied to models, and we extend them by analyzing in more details the differences between the responses to natural movies and spontaneous activity and by analyzing LFPs from anaesthetized macaques collected in separate experiments.

Fig. 1A shows single-trial traces of the delta and gamma frequency bands of the EEG signal recorded over visual cortex of an awake monkey performing a fixation task during the presentation of a colour naturalistic movie. The delta and gamma band EEG traces were obtained by bandpassing the single trial traces of the raw signal from the EEG electrode (see Methods) in the [2-4 Hz] and [30-100 Hz] frequency range, respectively. Comparison with the simultaneously measured spike trains from visual cortex (Fig. 1C) suggests that modulations of EEG gamma amplitude are clearly associated with modulation of the spike rate: during time periods with high gammaband EEG amplitude there is typically an increase of spike rate with respect to periods when the gamma oscillations have weak amplitude. A similar relationship between spike rate and gamma amplitude can be observed in the concurrent LFP traces (Fig. 1B). Moreover, visual 
A

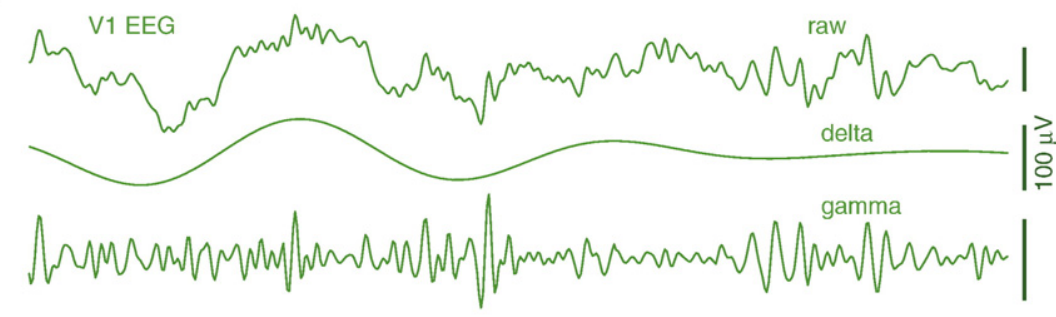

B

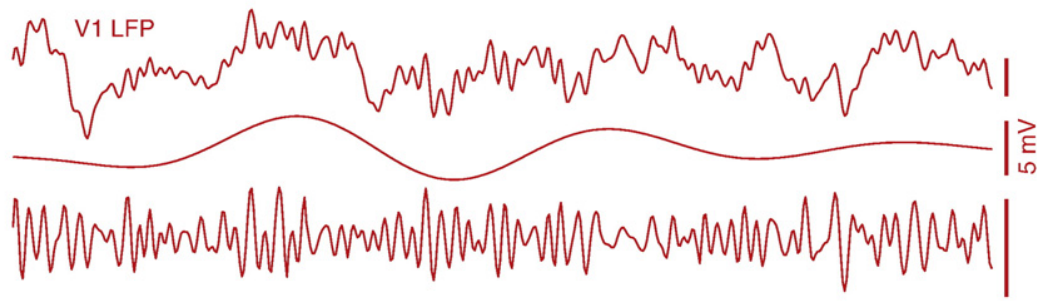

C

V1 SPIKES

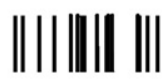

$\|$

$200 \mathrm{~ms}$

Fig. 1. Illustration of the time course of simultaneously recorded raw and band-passed EEGs and LFPs and spike rates during movie presentation. All signals were recorded simultaneously in the primary visual cortex of awake monkey during presentation of a colour movie. (A) EEG single-trial traces. Green lines indicate the raw signal (top), and the same signal band-passed in the 2- to 4-Hz delta band (middle), and in the 30- to 100-Hz gamma band (bottom). Note that each band-passed signal is scaled in a different way, and the scale of each signal is given by a vertical bar on the right hand side. (B) LFP single-trial traces. Red lines indicate the raw signal (top), and the same signal band-passed in the delta (middle) and gamma (bottom) bands respectively. Note that each band-passed signal is scaled in a different way, and the scale of each signal is given by a vertical bar on the right hand side. (C) Spiking activity. Vertical black lines indicate spike times.

inspection of EEG and LFP traces in Fig. 1 suggests that spike rate was higher during troughs of the EEG and LFP delta band, and was lower at its peaks.

The previous illustration based on a single trace is confirmed by a quantitative population analysis, which we performed as follows. We divided the time range into 2-ms bins. In each time bin and each trial, we computed the spike rate and also the associated instantaneous phase of the delta-band EEG or LFP and the amplitude of the gamma band EEG or LFP. Instantaneous amplitude and phase of each bandpassed signal were computed in each trial as the modulus and angle of the Hilbert transform of the band-passed signal, respectively. In Fig. $2 \mathrm{~A}$ we plot the spike rate as function of the LFP gamma amplitude and delta phase, averaged over all recording sessions, trials and time points during movie presentation. Spike rate was normalized between maximum and minimum value for each session before taking the average across sessions. Fig. 2A confirms that the LFP gamma amplitude was clearly correlated with spiking activity: on average, the higher was the gamma amplitude, the higher was the spike rate (Pearson correlation $r=0.26 ; p<0.001$ ). However, gamma amplitude alone did not entirely predict the spike rate because the phase of the delta band also modulated the spike rate (circular-linear correlation $r=0.12, p<0.001$ ), as well as the gamma amplitude (circular-linear correlation $r=0.08 ; p<0.001)$. Fig. $2 \mathrm{~A}$ shows that spike rate was maximal at the preferred phase pi and minimal at the anti-preferred phase 0 . Noteworthy, LFP delta phase and LFP gamma amplitude exerted a partly complementary role in predicting whether spike rates were high or low. In fact, Fig. $2 \mathrm{~A}$ shows that strong gamma responses occurring during delta peaks (phase 0 ) corresponded to a reduced spike rate compared to when an equally strong gamma response occurring during a delta trough (phase pi). Moreover, at any given LFP delta phase higher spike rates were achieved if the concurrent gamma oscillation had a higher amplitude. During movie stimulation sessions (Fig. $2 \mathrm{~A}$ ) the highest spike rate range ( $>75 \%$ of the maximum spike rate value) was obtained only for values of gamma amplitude above 0.9 sd over the mean concurrent with a delta phase between 2 and 4.3 radians. In summary, the cross-frequency coupling between LFP gamma amplitude and delta phase was crucial to determine the amount of spiking activity: only the right combination of delta phase and gamma amplitude gave rise to pronounced spiking activity.

We also investigated the relationship between spike rate, LFP delta phase and LFP gamma amplitude during stimulus-free periods. The dependence of the spike rate on the LFP gamma amplitude and delta phase during spontaneous activity (Fig. 2B) was very similar to the one obtained from the same population during movie stimulation, suggesting that similar mechanisms of cross-frequency modulation of spiking activity are at work even in absence of stimuli. Also during spontaneous activity spike rate correlated significantly to gamma amplitude (Pearson correlation $r=0.22 ; p<0.001$ ) and also to delta phase (circular-linear correlation $r=0.12 ; p<0.001$ ), and delta phase significantly modulated gamma amplitude (circular-linear correlation $r=0.11 ; p<0.001$ ). The most notable difference that we found between stimulus free and movie driven activity was that the tuning of spike rates to delta phase and gamma amplitude was sharper during spontaneous activity. During spontaneous activity sessions (Fig. 2B) the highest spike rates ( $>75 \%$ of the maximum spike rate) were associated only to gamma values above 2.1 sd over the mean concurrent with a delta phase between 2.7 and 3.7 radians, a much narrower set of gamma amplitude and delta phases values than the one found to give highest spike rates for the movie condition.

We then considered the relationships between spike rate and the delta phase and gamma amplitude of EEGs (rather than LFPs). Results are reported in Figs. $2 \mathrm{C}$ and D for movie-driven and stimulus-free activity respectively. Again, in both stimulation conditions, the same cross-frequency coupling mechanism (referred to as frequency-band coupling in Whittingstall and Logothetis, 2009) observed for LFPs described the relationship between the EEG oscillations and the spike rate. Spike rate was significantly correlated to EEG gamma amplitude (Pearson correlation $r=0.16$ and $r=0.20$ for movie and spontaneous condition, respectively, both significant at $p<0.001$ ) and also to EEG delta phase (circular-linear correlation $r=0.04$ and $r=0.06$ for movie and spontaneous condition respectively, both significant at $p<0.001$ ). Delta phase significantly modulated gamma amplitude 
A

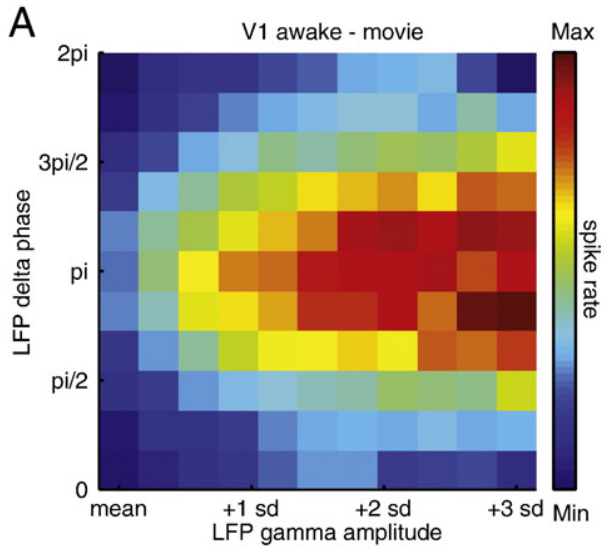

C

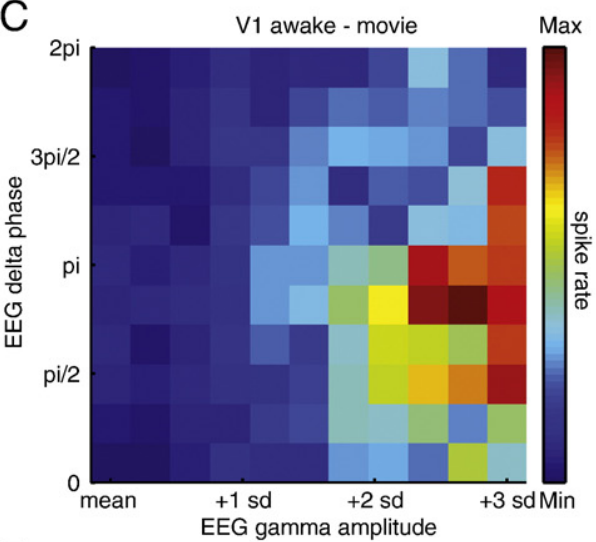

E

V1 anaesth. - movie

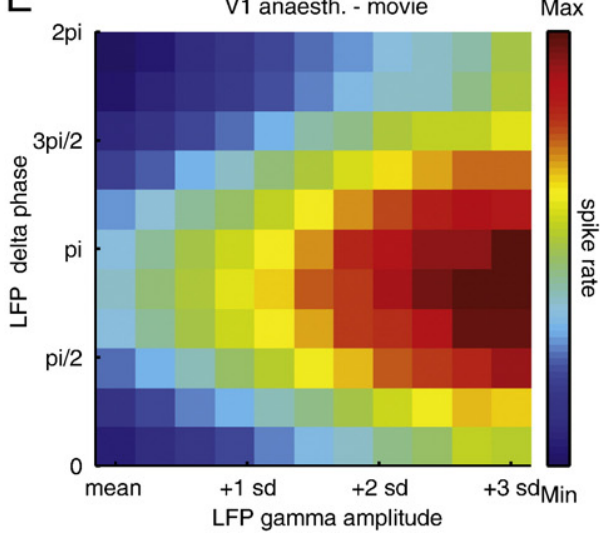

B

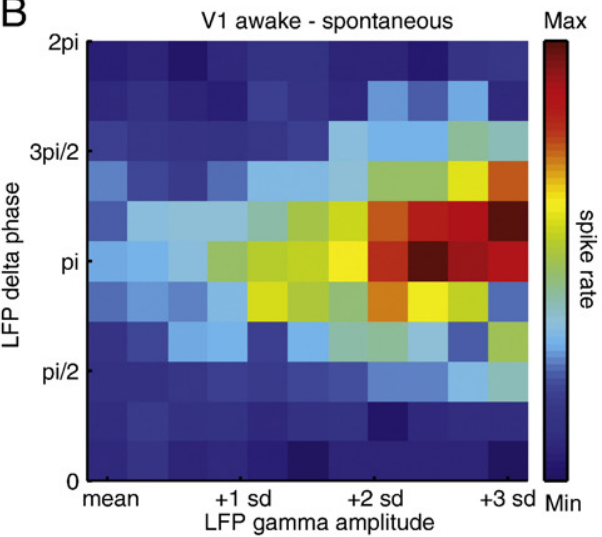

D

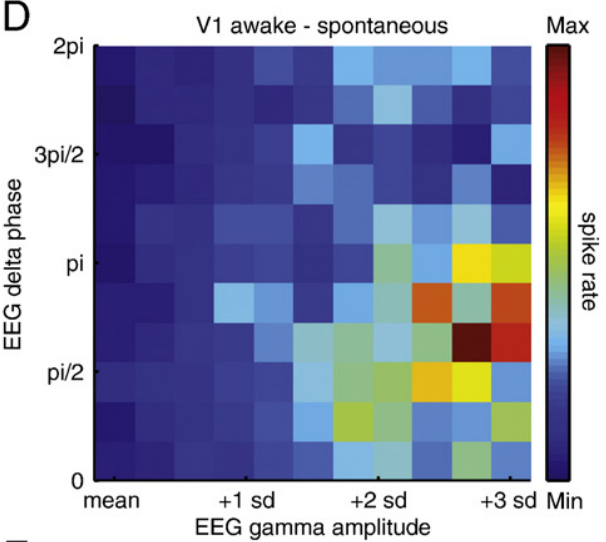

F

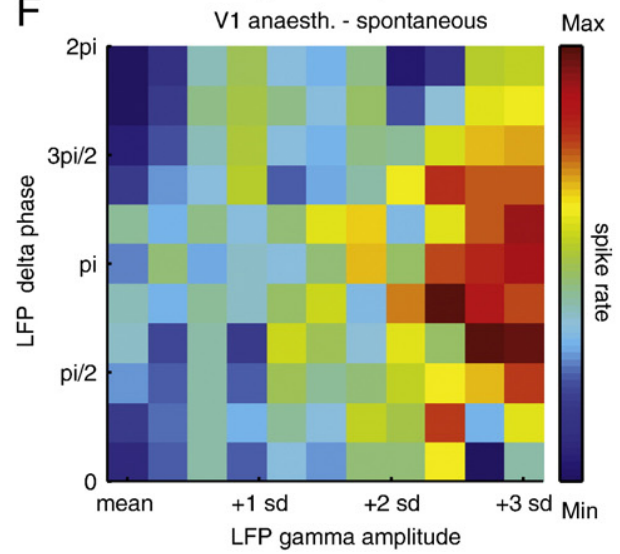

Fig. 2. Dependence of spike rate by the simultaneously observed gamma amplitude and delta phase of EEGs or LFPs in different experimental conditions. All plots report the spike rate as a function of the concurrent delta phase of and gamma amplitude of either LFPs or EEGs. Data were averaged over all time points, trials and recording sessions. The firing rate was normalized between minimum and maximum of each session before taking the average over sessions. The gamma amplitude was normalized in units of standard deviation (sd) within the session (above the mean across the session) before taking the session average. Panel A uses LFP and spike data recorded from awake monkeys during movie presentations. Panel B uses LFP and spike data recorded from awake monkeys during spontaneous activity. Panel C uses EEG and spike data recorded from awake monkeys during movie presentations. Panel D uses EEG and spike data recorded from awake monkeys during spontaneous activity. Panel E uses LFP and spike data recorded from anaesthetized monkeys during movie presentations. Panel F uses LFP and spike data recorded from anaesthetized monkeys during spontaneous activity.

(circular-linear correlation $r=0.045$ and $r=0.065$ for movie and spontaneous condition respectively, both significant at $p<0.05$ ). The main differences between the results with LFPs and EEGs was that only periods of very high EEG gamma amplitude (2 sd above the mean) modulated the spike rate in both movie and spontaneous condition, and that the preferred EEG delta phase of firing and gamma amplitude was slightly advanced with respect to the LFP case.

Extending the results of Whittingstall and Logothetis (2009) for the awake monkey, we repeated the same analysis on LFP recordings from primary visual cortex of anaesthetized macaques. We found a very similar pattern of dependency between the spike rate and the LFP delta phase and gamma amplitude (Figs. 2E and F), both during movie stimulation and spontaneous activity. Spike rate was again significantly correlated to gamma amplitude (Pearson correlation $r=0.06$ and $r=0.04$ for movie and spontaneous condition respectively, both significant at $p<0.001$ ) and also to delta phase (circular-linear correlation $r=0.04$ and $r=0.02$ respectively for both movie and spontaneous condition, both significant at $p<0.001$ ). Delta phase significantly modulated gamma amplitude (circular-linear correlation $r=0.03$ and $r=0.04$ for movie and spontaneous condition, significant at $p<0.001$ ). Similarly to what was found with awake monkeys, the highest spike rates ( $>75 \%$ of the maximum rate) were found in correspondence with values of gamma amplitude above 0.9 sd over the mean combined with a delta phase between 0.9 and 4.3 in 
the movie condition and in correspondence with values of gamma amplitude above 1.8 sd over the mean combined with a delta phase between 1.5 and 4.5 radians in the spontaneous condition. This consistency is important both because it shows the robustness of the phenomenon, and because these anaesthetized data will later be used for constructing the model.

We note that we reported results of the relationship between spike rate and the phase of the delta band and the amplitude of the gamma band because (both during stimulation and during spontaneous activity) the gamma band was the only band whose amplitude correlated strongly with the spike rate (results not shown; but see (Belitski et al., 2008; Rasch et al., 2008; Whittingstall and Logothetis, 2009)), and the delta band was the one whose phase provided the strongest modulation of spike rate, the latter decreasing fast with the frequency at which the phase was considered (results not shown; but see Montemurro et al., 2008; Whittingstall and Logothetis, 2009).

\section{Neural network model-hypothesis and structure}

To explain the above empirically measured dependence of the instantaneous spike rate on the EEG and LFP phase and amplitude, here we make the hypothesis that the EEG-LFP delta phase reflects shifts of the cortical excitability arising from slow (delta range) fluctuations in the strength of the input to the local network, and that these changes in excitability modulate both the output spike rate and the engagement of excitatory-inhibitory loops producing gammaband oscillations, which in turn leads to the three-way relationships between spike rate, gamma amplitude and delta phase.

To quantitatively test this hypothesis, we simulated a recurrent network of spiking neurons with excitatory-inhibitory connections stimulated with dynamic inputs, similar to the one used in Brunel and Wang (2003) and Mazzoni et al. (2008). In brief (see Methods for full details), the model network represented in a simplified way a recurrent local circuit in primary visual cortex, and was a recurrent network with sparse random connectivity made of integrate-and-fire neurons representing inhibitory interneurons and pyramidal neurons (a schematics is plotted in Fig. 3A). Synaptic currents represented fast synaptic interactions, with time courses resembling experimentally measured AMPA currents (for excitatory currents) and GABA currents (for inhibitory currents). The strength of GABAergic connections was sufficient to ensure stable activity at low spike rates in the network. Both populations received an excitatory external input taken to represent the activity from thalamocortical afferents, with interneurons receiving stronger inputs than pyramidal neurons (Gil and Amitai, 1996), and an additional excitatory external input taken to represent the activity from non-local cortical afferents carrying
A

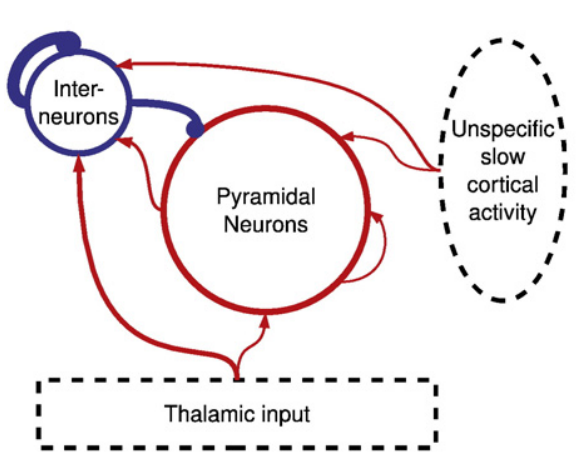

C

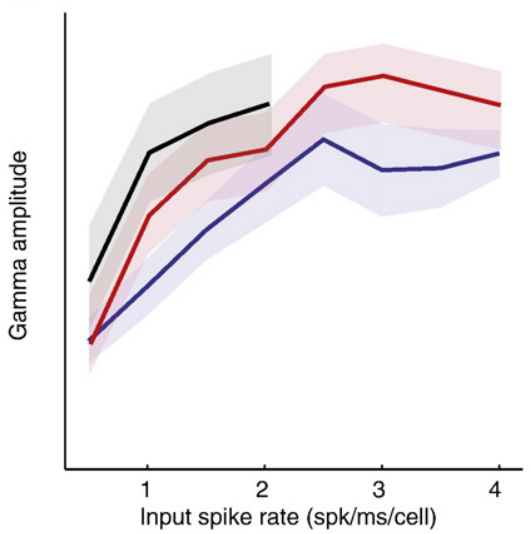

B

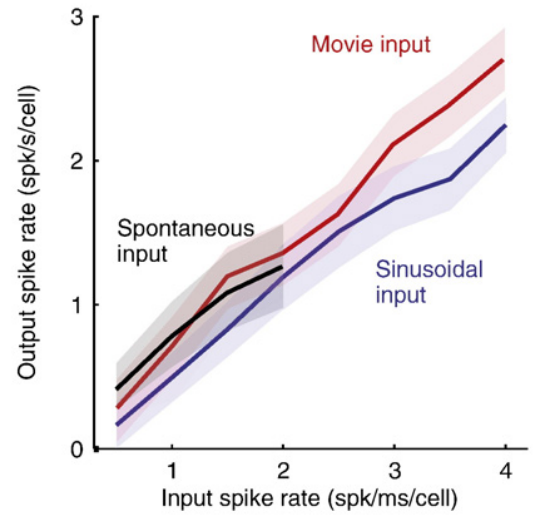

D

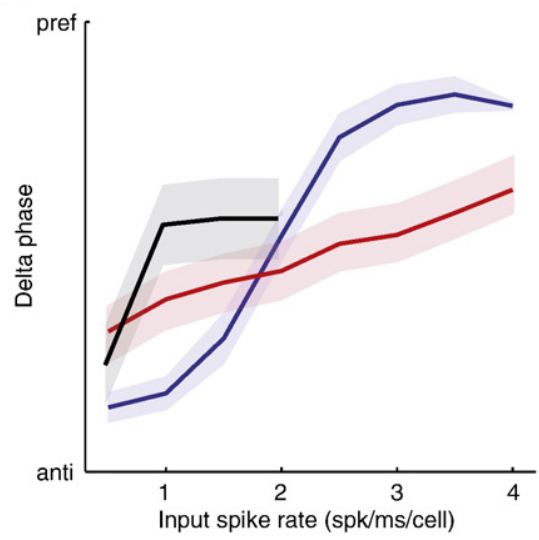

Fig. 3. Structure and input-output relationships of the simulated local cortical model. (A) Schematic of network structure. The network is composed by 4000 pyramidal neurons and 1000 interneurons. The connectivity is random, a synapse being present between any directed pair of neurons with probability 0.2 . The size of the arrows represents schematically the strength of single synapses: recurrent interactions are dominated by inhibition. In addition to recurrent interactions each neuron receives excitatory inputs from the thalamus and from non-local and non-specific ongoing cortical activity. (B) Average spike rate of model pyramidal neurons in response to different values of the instantaneous input spike rate. Lines represent the mean over all 10-ms time windows in the simulations (with sd represented as shaded area). The red and the black line refer to data obtained when using as inputs LGN activity recorded during movie stimulation or spontaneous activity respectively. Spontaneous input rate never exceeds 2 spikes/ms/cell. The blue line refers to data obtained using a sinusoidal input with same average of the LGN movie input, $2 \mathrm{~Hz}$ frequency and $0.8 \mathrm{spikes} / \mathrm{ms} /$ cell amplitude. (C) Gamma amplitude of the LFP of the network for different values of the input spike rate, with conventions as in (B). The simulated gamma amplitude is expressed on an arbitrary scale which is however the same for all conditions and input rate values. (D) Delta phase of the LFP of the network for different values of the input spike rate, with lines colour coded as in (B). Phase was normalized as ranging from anti-preferred to preferred (the ones eliciting the lowest and highest probability of firing on average). In this panel, lines represent the circular mean of the delta phase and areas its circular standard deviation. 
activity not specific to the particular stimulus shown. Simulated LFPsEEGs from the network were computed as the sum of the absolute values of synaptic currents from synapses of the pyramidal neurons population (see Methods for a complete description). This is because a prominent contribution to both LFPs and EEGs comes from synaptic activity in apical dendrites of pyramidal neurons. In our simple model we did not to distinguish between simulated EEGs and simulated LFPs. This is of course an oversimplification, because EEG and LFPs are signal which share some similarities but also many differences (for example, EEGs have a much coarser spatial resolution and their higher frequencies are attenuated by the skull). However, the EEG and LFP recordings under naturalistic movie stimulation that we analyzed were very consistent (see Figs. 1 and 2), suggesting that in these stimulation conditions a large area was activated in a consistent way, probably due to the spatial and temporal regularities of the naturalistic stimulus and to the spatially extended nature of spontaneous fluctuations. Further, our model was able to replicate the basic relationship between spiking activity and both LFPs and EEGs, confirming that it could capture the basic features of both signals under the specific experimental conditions examined here.

The dynamical input-output properties of the network can account for cross-frequency modulation of instantaneous spike rates

To gain insights into the mechanisms driving the relationship between the coupling of oscillations and spiking activity in these networks, we began by studying how the network behaves when stimulated with simple inputs, which are either constant in time with a given mean rate, or inputs which vary sinusoidally in time at a given frequency. As we will see, the understanding gained in these simple conditions will be helpful to understand the network responses to complex broadband inputs such as the inputs to cortex during stimulation with naturalistic movies or during spontaneous activity.

We first investigated the relationship between the strength of the input spike rate at a given time and the corresponding network output spike rate (which was taken by summing the instantaneous spike rate of all simulated excitatory neuron, because this is comparable to what would be measured experimentally by placing an extracellular electrode in visual cortex (Logothetis, 2003)). Fig. 3B (blue line) shows that in case of a simple sinusoidal input the network output rate was roughly proportional to the input rate, consistent with previous studies of recurrent networks of excitatory and inhibitory neurons in the balanced regime (Brunel, 2000; van Vreeswijk and Sompolinsky, 1996, 1998). This finding shows that the network output rate (corresponding to the one measured by electrodes in V1) is proportional to the input to the network, and therefore the relationship between measured spiking activity and LFPs and EEGs can be understood in the simulated network by characterizing the relationship between the input rate and the EEG and LFP fluctuations.

The most prominent dynamic feature of the network is that it generates fast oscillations in the gamma [30-100 Hz] range, as widely documented in previous studies e.g. (Brunel and Wang, 2003; Mazzoni et al., 2008). Two features of the recurrent connectivity contribute to the generation of network oscillations: (1) delayed interactions between interneurons and (2) the excitatory-inhibitory feedback loops. One crucial property of such excitatory-inhibitory recurrent networks is that the strength of the population oscillation strongly depends on external inputs to the network (Brunel and Wang, 2003). Typically, for low enough external inputs, the network is in an asynchronous state, with weak and strongly damped oscillations, while for strong external inputs, the network tends to settle in a pronounced oscillatory state. This is illustrated in our case in Figs. 4A, B, which shows that time-independent external stimuli to the network generate gamma-range LFP responses whose amplitude and frequency grows with the input rate (see (Brunel and Wang, 2003) for a systematic analysis). The fact that both gamma amplitude and output spike rate of the network are largely determined by the input rate explains why gamma amplitude can be used to estimate the output spike rate. However, it is important to note that we found (Fig. 3C) that the conversion between input rate and gamma amplitude is non-linear, as the gamma amplitude tends to increase almost linearly with the input rate for small values of the latter, whereas it rapidly saturates to a sublinear growth for higher values of the input rate. The saturation of gamma amplitude at high rates has important implications for the problem of estimating spike rates from EEGs and LFPs, because it suggests that gamma amplitude variations may better predict variations in spike rates associated with low levels of input excitation rather than at very high levels of excitation.

When periodic external stimuli with frequency lower than that of gamma oscillations are presented, the network still generates gamma fluctuations whose amplitude is proportional to the instantaneous input rate (see Figs. 4C, D for an illustration, and Fig. 3C for a quantitative analysis averaged over simulations). However, in addition, the network generates slower oscillations (Figs. 4C, F) which reflect entrainment of the network to the time course of the external stimulus. These slow oscillations reflecting entrainment to the variations in the input are clearly visible in the accompanying LFP and in the output rate (see Figs. 4C, F for single-trace examples and Fig. 3D for a quantitative analysis averaged over simulations). In this case, troughs of the LFP reflect local maxima of the input rate, and thus also correspond to peaks of the network output rate and of the gamma amplitude of LFP (Figs. 4C, F). Thus, when a slow LFP component reflects entrainment to the fluctuations of the input rate, the phase of such slow LFP rhythm will modulate both the output spike rate and the gamma amplitude, in a way resembling that observed in visual (Montemurro et al., 2008; Rasch et al., 2008; Whittingstall and Logothetis, 2009) and auditory (Lakatos et al., 2005) cortex.

These considerations can also help to understand the experimental findings that the combination of EEG or LFP gamma amplitude and delta phase can predict the output spike rate better than any of the two variables in isolation. The fact that delta phase alone cannot predict the rate as well as when delta phase and gamma amplitude are put together can be explained by taking into account that, under such entrainment conditions, the delta phase reflects whether the shift in input excitation reaches a maximum or minimum over time, but does not tell the actual strength of the input at a given time. Therefore, in cases when there is a strong modulation of instantaneous input amplitude, delta phase will be a poor predictor compared to the gamma amplitude. This is illustrated by Figs. 4E, F. When the periodic input has no temporal variations in amplitude (Fig. 4E) delta phase can be used to predict spike rate because spike rate is equally high in all LFP delta troughs. However, when the periodic input has strong variations in amplitude (Fig. 4F), then delta phase cannot predict any more the actual spike rate because spike rate is very different in each LFP delta trough. In this case, gamma amplitude is the better predictor of spike rate.

Conversely, the saturation of gamma amplitude at high input rate (Fig. 3C) explains also why in some circumstances delta phase can give information about the spike rate not achievable by gamma amplitude. If the network operates at most times in a high input regime (such as the case of an oscillating input with high baseline firing and small amplitude variations over time; Fig. $4 \mathrm{H}$ ), then the gamma amplitude is almost constant in time and (unlike delta phase) does not predict the spike rate as well as it does when the network has the same amplitude variations over time but a smaller baseline and so does not operate in the gamma amplitude saturation regime (Fig. 4G)

In summary, when the LFP or EEG delta phase reflects changes in the network excitability entrained to periodic variations in the network input, its phase will also modulate the output spike rate and will modulate the LFP and EEG gamma amplitude. Moreover, because of nonlinearities in the conversion between the input rate to the network and the gamma amplitude generated by the network, and whenever there are significant modulations over time of the 
A
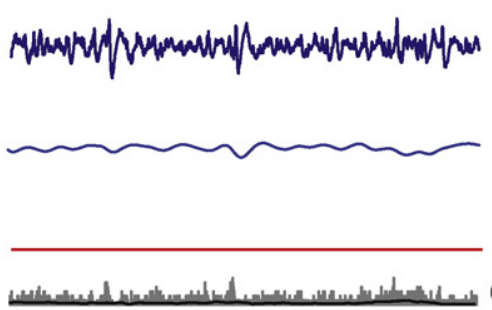

C

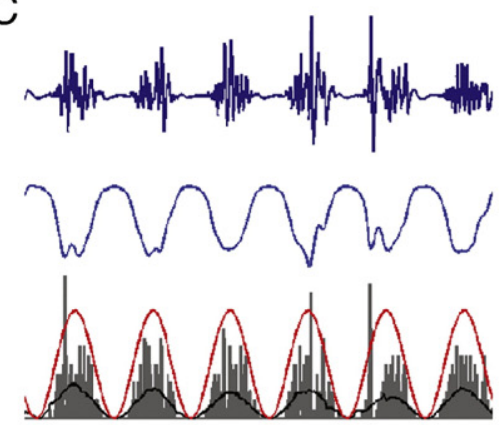

E

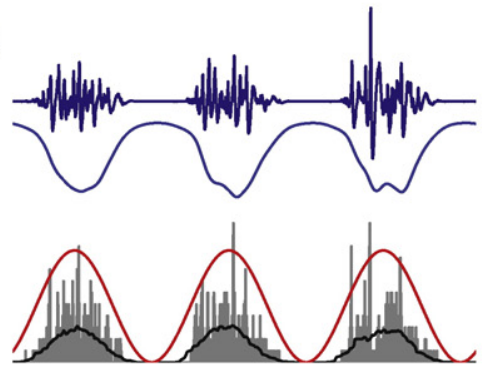

G

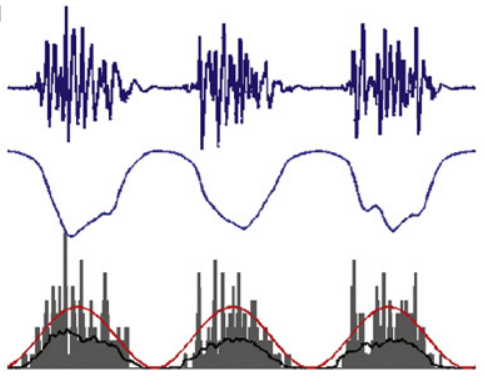

B

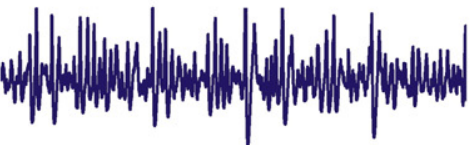

gamma LFP/EEG

delta

LFP/EEG

Inp. spk rate

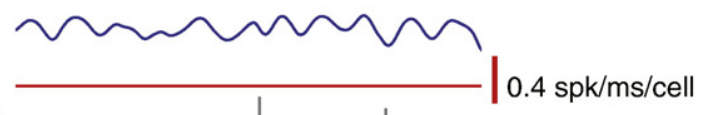

$0.4 \mathrm{spk} / \mathrm{ms} / \mathrm{cell}$

$2 \mathrm{spk} / \mathrm{s} / \mathrm{cell}$

D
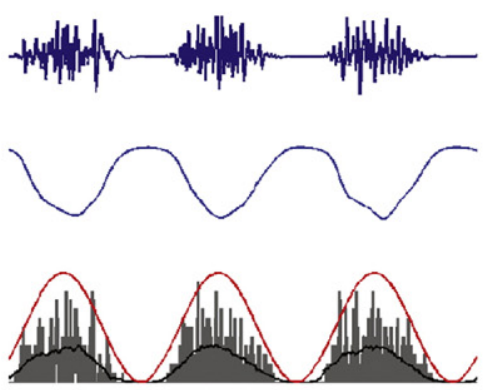

$\mathrm{F}$

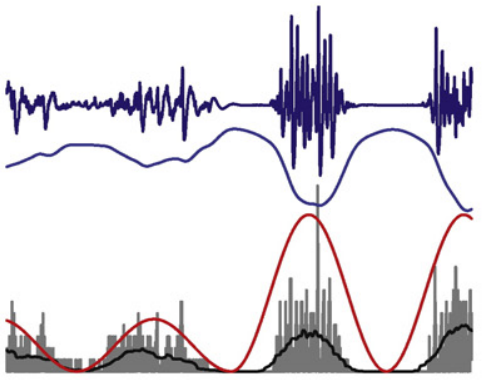

$\mathrm{H}$

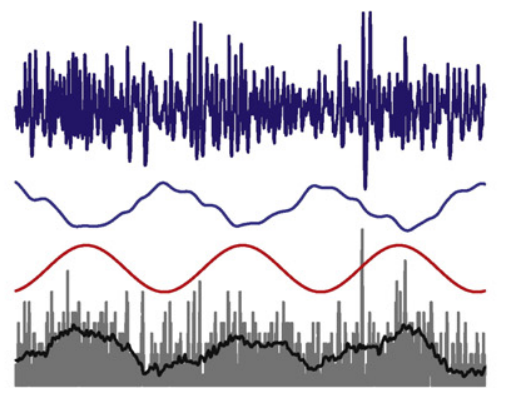

Fig. 4. Examples of network responses to simple time-dependent thalamic inputs. Each panel displays the time series of single-trial simulated traces of gamma EEG/LFP band activity (top dark-blue line), delta EEG/LFP band activity total spike rate (computed with 1 ms bin-gray line, and smoothed at 100 ms-black line) produced by the simulated network in response to a thalamic simulated spike rate whose time course is displayed as the red line (the value of the instantaneous thalamic input rate is represented by the height of the red line measured form the bottom of the panel). The traces of simulated EEG/LFP bandpassed signals are plotted on an arbitrary scale which is however the same in all cases. (A) Timeindependent input of $0.4 \mathrm{spikes} / \mathrm{ms} /$ cell. (B) Time-independent input of 0.8 spikes $/ \mathrm{ms} /$ cell. The increase in input spike rate with respect to Panel (A) leads to enhanced gamma amplitude. (C) Sinusoidal input with $4 \mathrm{~Hz}$ frequency, 0.8 spikes $/ \mathrm{ms} /$ cell amplitude. The network LFP is entrained to the input, as in all the following panels. (D, E) Sinusoidal input with $2 \mathrm{~Hz}$ frequency, 0.8 spikes/ms/cell amplitude. (F) Sinusoidal input with $2 \mathrm{~Hz}$ frequency, amplitude of $0.4 \mathrm{spikes} / \mathrm{ms} / \mathrm{cell}$ (left) and $1.2 \mathrm{spikes} / \mathrm{ms} / \mathrm{cell}$ (right). Due to the change in amplitude, different output spike rates correspond to the same delta phase. (G) Sinusoidal input with $2 \mathrm{~Hz}$ frequency, $0.4 \mathrm{spikes} / \mathrm{ms} / \mathrm{cell}$ amplitude. (H) Sinusoidal input with $2 \mathrm{~Hz}$ frequency, 0.4 spikes $/ \mathrm{ms} /$ cell amplitude, and a +0.8 spikes $/ \mathrm{ms} /$ cell constant term added. Due to the high input spike rate, the LFP gamma amplitude correlates weakly to the output spike rate.

amplitude of the delta-band input fluctuations, gamma amplitude and delta phase are expected to have a largely complementary correlation with the output spike rate, meaning that only the appropriate combination of delta phase and gamma amplitude will correlate to very strong spike rates.

Behaviour of the network during naturalistic and spontaneous stimulation

The above results suggest that a dynamic input with a slow periodic component is able to entrain the simulated network and generate relationships of modulation of gamma amplitude and spike rate by the phase of slow input changes which are very similar to those observed experimentally with respect to the phase of LFPs and EEGs in the delta frequency range. It is therefore tempting to speculate that the relationships between delta phase and gamma amplitude and spike rate observed in visual cortex (Whittingstall and Logothetis, 2009) originate because the input to the local primary cortical population exhibits the largest temporal variations at temporal scales roughly in the delta frequency range, both during stimulation with 
naturalistic movies and in absence of stimuli. Indeed, naturalistic movies (because of strong low-frequency regularities) have most power in the delta frequency range (Dong and Atick, 1995), and it is thus likely that (as a result of the stimulus dynamics) thalamic responses during naturalistic stimulation will also present response fluctuations with most power in this range. This view is consistent with the finding that presentation of naturalistic movies induce highly informative slow (1-8 Hz) LFP fluctuations in V1 (Belitski et al., 2008; Montemurro et al., 2008), which were not significantly modulated and informative with static stimuli (Henrie and Shapley, 2005), and with the finding that stimuli with temporal regularities in this frequency range easily entrain LFPs and network firing (Lakatos et al., 2008; Lakatos et al., 2005). Moreover, both during spontaneous activity and during visual stimulation, a local cortical network is also likely to receive inputs from other cortical areas which reflect ongoing activity fluctuations and are not specific to the current stimulus. It is conceivable that such input contributions from ongoing cortical activity are more prominent in the slow (delta) frequency range than at higher frequencies given that spontaneous shifts of excitability also occur in the slow frequency range (Contreras and Steriade, 1995; Luczak et al., 2009; Luczak et al., 2007), and because low-frequency cortical fluctuations have a larger spatial coherence than faster fluctuations (Goense and Logothetis, 2008) and so their effect on other areas may summate more prominently than that of faster fluctuations.

To investigate these hypotheses more quantitatively, we simulated these phenomena within our model network (Fig. 3A). We created an input to the network closely matching the statistics of thalamic firing during visual stimulation with natural movies (called "naturalistic movie input" in the following) injecting into the network real LGN firing patterns recorded during presentation of movies (see Methods). We created the spontaneous stimulus-unrelated fluctuations by injecting into the simulated thalamocortical synapses the LGN firing patterns recorded during spontaneous activity (this will be called "spontaneous activity input" in the following). In both conditions the thalamic input was superimposed to an input from non-local cortical neurons, injected into the cortico-cortical synapses spikes driven by slow random fluctuations with most power below $10 \mathrm{~Hz}$ (see Methods), which modelled the effect of ongoing non-specific spatially extended fluctuations of cortical activity (see above). It was apparent (Supplemental Fig. S2) that also when using such broadband inputs the low frequency components of simulated LFPs/EEGs entrained to the peaks and troughs of the simulated thalamic input, both during naturalistic movie input and spontaneous activity input. In the following we investigated quantitatively if this entrainment to slow input fluctuations could reproduce in the simulated network the details of the experimentally observed relationships between spike rate, gamma amplitude and delta phase.

We first investigated the spectral properties of the simulated thalamic input during movie stimulation and during spontaneous activity. The power spectrum of the simulated input spike train in both conditions had the highest power in the low frequency range, and the LFP in the delta range entrained to fluctuations in the input on the same scales (Supplemental Fig. S3). This is consistent with our own previous measures of power of the temporal frequencies of contrast variations in the very same movies used in the experimental sessions and reported in Belitski et al. (2008) and Montemurro et al. (2008). Moreover, there was more overall power in the input during naturalistic movie condition, compatible with the fact that on average the spike rates recorded in the monkey LGN were higher during movie presentation than during spontaneous activity ( $17 \pm 6$ and $9 \pm 4$ spikes/s per recording channel respectively).

We then studied the relationship between the instantaneous level of input spike rate and the associated network response during simulated "naturalistic movie" stimulation. We found that, as with simplified input stimuli, the output spike rate remained proportional to the input rate (Fig. 3B), and the gamma amplitude increased with the input spike rate and saturated at higher input rates (Fig. 3C). The input rate modulated the delta phase too (Fig. 3D). In the naturalistic case the tuning of delta phase to input rate was more linear and more limited in range than in the sinusoidal case (Fig. 3D), probably because of the modulations in frequency and instantaneous amplitude occurring in the naturalistic movie. All in all, these results suggest that the modulation mechanisms described with simple inputs also hold when presenting broadband naturalistic inputs.

We then investigated whether the network could reproduce quantitatively the dependency of spike rates on gamma amplitude and delta phase of EEGs and LFPs observed in primate V1 during presentation of naturalistic movies and spontaneous activity. Figs. $5 \mathrm{~A}$ and $B$ reports the output spike rate of the simulated network as function of the concurrent simulated LFP delta phase and gamma amplitude, and demonstrates that the network reproduces very well the results obtained in monkey V1 and previously summarized in Fig 2, both with movie stimulation and spontaneous activity. In particular, also in simulations only the right combination of delta phase at trough and high enough gamma amplitude elicited a high output spike rate. Similarly to experimental results, the output spike rate was significantly correlated both to gamma amplitude (Pearson correlation $r=0.14$ and $r=0.22$ respectively for movie and spontaneous condition, both significant at $p<0.001$ ) and to delta phase (Figs. 5C, $\mathrm{D}$, circular-linear correlation $r=0.11$ for both movie and spontaneous condition; $p<0.001)$. Delta phase significantly modulated gamma amplitude (Figs. 5E, F, circular-linear correlation $r=0.17$ for both movie and spontaneous condition, significant at $p<0.001$ ). Moreover, the simulated network's tuning of gamma amplitude to spike rates was narrower during spontaneous activity than during movies, exactly as observed in real data. During movie input sessions (Fig. 5A) the highest spike rates ( $>75 \%$ of the maximum spike rate) occurred in correspondence of gamma amplitude values above the mean gamma amplitude value combined to a delta phase between 2 and 4.3 radians. During spontaneous input sessions (Fig. 5B) the highest spike rates were associated to the same delta phase range as with the movie stimulus, but occurred only at higher gamma amplitude values ( $0.9 \mathrm{sds}$ above the mean) than in the movie condition. This difference can be understood within the model by noting that (as shown in Fig. 3C) the spontaneous simulated input does not contain input spike rates as strong as those with the simulated naturalistic movies, and thus the network operates more in the low-rate linear regime of input rate to gamma amplitude conversion. This is a possible explanation for the difference in the dependence of spike rate upon gamma amplitude and delta phase between experimental movie and spontaneous condition shown in Fig. 2.

\section{Robustness to parameters changes of the behaviour of the simulated network}

The relationships between spike rate and delta/gamma frequency bands of a mesoscopic signal based on network activity are expected to hold qualitatively in any network of spiking neurons, provided the following conditions are satisfied: (i) the temporal structure of the inputs to the network (whatever their origin) should contain significant fluctuations in the delta frequency range (ii) the connectivity of the network is such that the network is able to generate population oscillations in the gamma frequency range when the external drive varies within the dynamic range characterizing the timedependent input to the network. Theoretical work in the last decade has shown that condition (ii) is generically satisfied in randomly connected networks of excitatory and inhibitory spiking neurons, provided that the coupling is strong enough, that recurrent interactions are dominated by inhibition, and that synapses have appropriate kinetics. In these networks, strong coupling leads to a balance between total excitatory and inhibitory currents (Amit and Brunel, 1997; Brunel, 2000; van Vreeswijk and Sompolinsky, 1996, 1998). This balance leads 
A

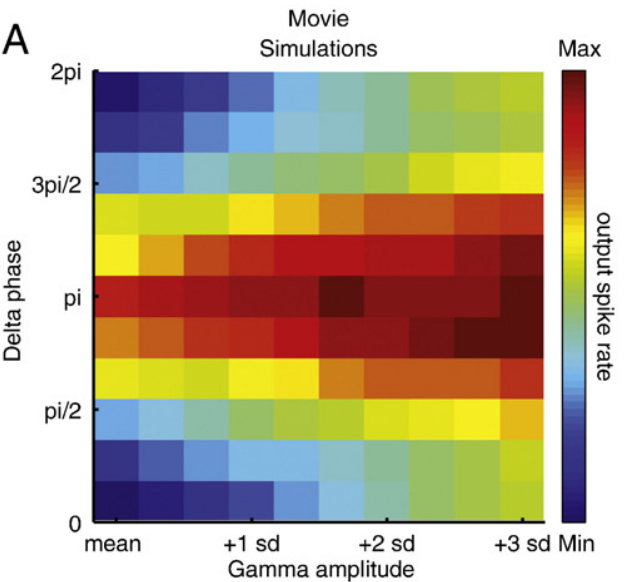

C

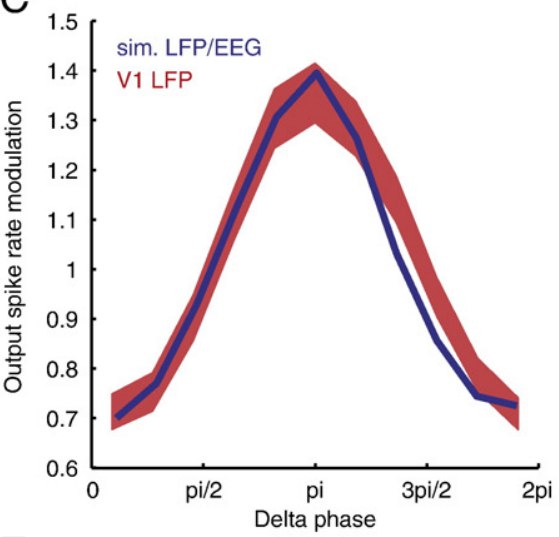

E

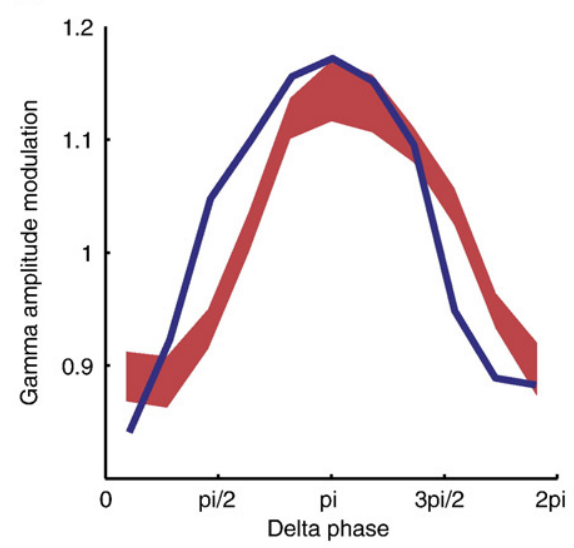

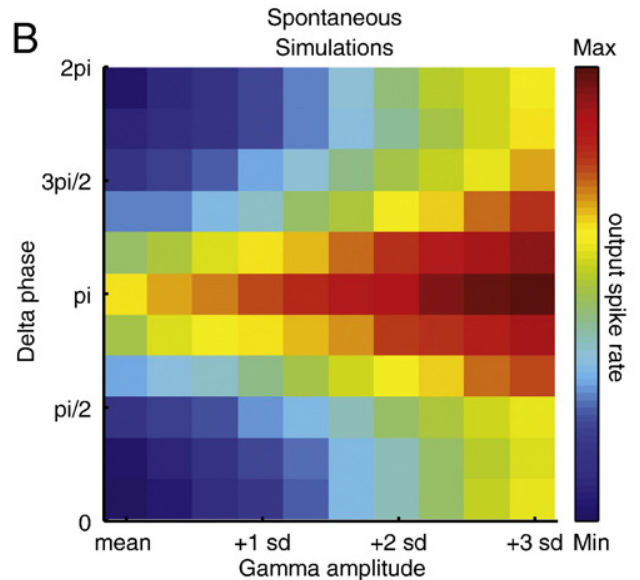

D

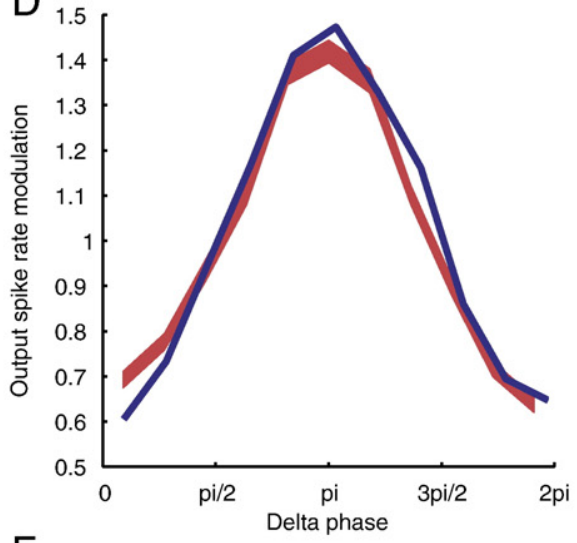

F

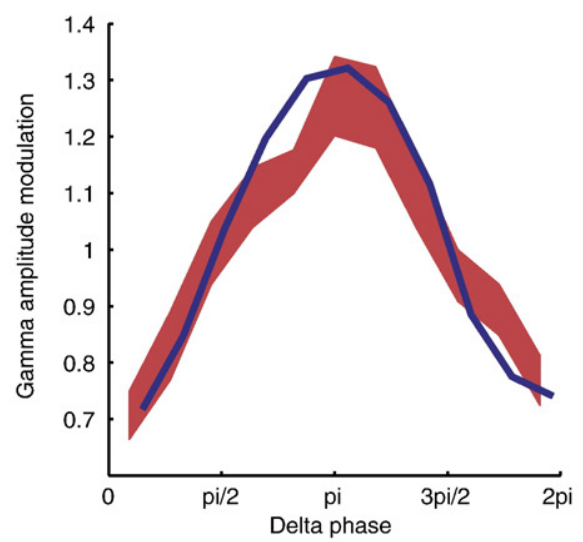

Fig. 5. Relationships between spike rates, delta phase and gamma amplitude produced by the network model in response to realistic thalamic inputs. (A) Color plot of the output spike rate of the simulated network as a function of the concurrent delta phase of and gamma amplitude of simulated LFPs/EEGs when the network responds to the thalamic input simulating realistic responses to movies. Data were averaged over all simulated time points. The spike rate was normalized between minimum and maximum of each simulation before taking the average over simulations, to allow a direct comparison with Figs. 2A, B. The gamma amplitude was normalized in units of standard deviation (sd) within the simulation (above the mean across the simulation) before taking the simulation average. (B) Same as panel A, but reporting simulated network responses when the network responds to the thalamic input simulating spontaneous activity. (C) The modulation of spike rates as a function of the concurrent LFP delta phase recorded from V1 of awake monkeys (red shaded area represent the mean and SEM across recording sessions) is compared to the modulation of simulated network spike rates obtained in response to "naturalistic movie" simulated input as function of simulated LFP delta phase (blue lines; average over all simulation points). (D) Same as panel C, but the cortical and simulated data are obtained during spontaneous activity. (E) The modulation of LFP gamma amplitude as a function of LFP delta phase recorded from V1 of awake monkeys (red line and shaded area represent the mean and SEM across recording sessions) is compared to the modulation of simulated gamma LFP amplitude obtained in response to a "naturalistic movie" simulated input as function of simulated LFP delta phase (blue lines; average over all simulation points). (F) Same as in panel E, but the cortical and simulated data are obtained during spontaneous activity. In panels C-F, modulation of spike rate or gamma amplitude by delta phase was computed as follows. First, we binned into 11 equispaced intervals the range of delta phase angles from 0 to $2 \mathrm{pi}$, and then in each phase interval we computed the average spike rate or gamma amplitude across all data points in a given session whose phase belonged to that phase interval, and we finally normalized this value to the spike rate or gamma amplitude averaged over all data points in the session. The resulting values gave the modulation curves in panels $(\mathrm{C}-\mathrm{F})$.

to a quasi-linear relationship between external inputs and instantaneous network firing rate (Fig. 3B), which ensures that variations of external inputs lead to a wide range of instantaneous firing rates (Brunel, 2000; van Vreeswijk and Sompolinsky, 1996). In addition, these networks generate population oscillations, provided the external drive is strong enough (Brunel, 2000; Brunel and Wang, 2003; Geisler et al., 2005; Mazzoni et al., 2008). The frequency of such oscillations depends on synaptic kinetics and on the balance between recurrent 
excitation and recurrent inhibition, and is typically in the gamma range for realistic synaptic time constants, provided recurrent excitation is not too weak. The results have been shown to be robust to changes in single neuron models (Geisler et al., 2005), except that the frequency of fast oscillations are expected to be smaller when realistic spike generation is taken into account. However, these effects are expected to be small for gamma-range oscillations.

Based on the above considerations, we can gain some intuition on how the various parameters of the network will affect the results. Synaptic parameters are expected to have a stronger effect. Parameters affecting synaptic kinetics have a strong impact on network frequency. Sufficiently slow (respectively fast) synaptic kinetics will lead to slower (respectively faster) oscillations. Given that we used a definition of gamma band as the [30-100] Hz range, modifications of kinetic synaptic parameters that leave the dominant network oscillations in this frequency range are not expected to modify the results substantially. Synaptic conductances are expected to have a strong impact on oscillations amplitude: the stronger the coupling in the network, the stronger oscillations. In particular, gamma oscillations should become negligibly small if the coupling in the network is very weak. Connection probability will have a similar effect as synaptic conductances: if connectivity is too sparse, then oscillations will become negligible.

We verified that these intuitions numerically by modifying in our network AMPA and GABA synaptic strengths in the range $\pm 20 \%$, GABA and AMPA synaptic rise times in the range $\pm 100 \%$, connection probability in the range $\pm 25 \%$. We found that, though naturally those parameters changed the overall rate and the peak frequency within the range of gamma oscillations, when injected with movie inputs the network exhibited robust gamma oscillations in this parameter range and all the documented relationship between input rate, delta phase, gamma amplitude and output rate remained valid. Namely, (i) delta phase and output rate varied linearly with the input rate, while gamma amplitude varied sublinearly (Figs. 3B, D), (ii) output rate and gamma amplitude were modulated by delta phase in the way illustrated in Fig. 5. The most notable effect of parameter variation was that the preferred delta phase of firing slightly anticipated $(<\pi / 4)$ when the strength of excitation was increased and/or connection probability was increased. This is because in such conditions rising phases of the input tend to produce a quick growth in the activity of the excitatory population, which is later suppressed by inhibition.

Using insights from network models to increase the predictability of rate from EEGs

The relationships between EEGs and spike rate are useful for practical EEG analysis only if they ultimately lead to simple rules of prediction of the actual time course of the spike rate from the EEG measure. A linear and successful EEG-to-spike rate prediction rule, based on the relationships between spike rate, gamma amplitude and delta phase reported in Fig. 2, was developed by (Whittingstall and Logothetis, 2009). Here we investigate whether such linear prediction of spike rates also works with the simulated model, and whether it is possible to use the insights into the neural mechanisms of this relationship gained by the model to improve the prediction of spike rates from real EEGs and LFPs.

We used the procedures described in Whittingstall and Logothetis (2009) for reconstructing the spike rate $r$ from EEG with a linear regression model:

$r=\beta_{\delta} X_{\delta}+\beta_{\gamma} X_{\gamma}+k$

where $k$ is a constant term, the gamma amplitude regressor $X_{\gamma}$ is the gamma amplitude normalized to its peak value and the regressor of the delta phase $X_{\delta}$ is one minus the normalized angular difference between the current phase and the preferred one, therefore ranging from 0 (anti-preferred phase) to 1 (preferred phase). Both rate and regressors where convolved with a 100-ms Gaussian window, since we found that, consistent with Rasch et al. (2008), this was the timescale at which spike rates can be predicted from averaged extracellular signals. Coefficients $R^{2}$ for real EEG and LFP data from V1 of the awake macaque (Whittingstall and Logothetis, 2009) were significant for all recording sessions if either delta phase or gamma amplitude was used in isolation ( $F$ test; $p<0.01$ ), and the regression quality still significantly improved over that of single regression if both delta phase and gamma amplitude were used in the reconstruction ( $F$ test; $p<0.01$ ). Figs. $6 \mathrm{~A}$, B displays the $R^{2}$ coefficients for LFPEEG recorded during movie stimulation; coefficients for LFP-EEG recorded during spontaneous activity (not shown) have very similar rankings but are reduced on average of 30\% for LFP and of $40 \%$ for EEG recordings. Consistent results were found with the network model (Fig. 6C). Again, delta phase and gamma amplitude gave significant linear regression when used in isolation, and the quality of regression too significantly increased using both gamma amplitude and delta phase ( $F$ test; $p<0.01$ ). We note that the higher $R^{2}$ prediction values obtained with the model when compared to those of LFPs were due to the fact that the spike rate used by the model was the spike rate of all 4000 pyramidal neurons in the network, whereas the estimation of spike rate on real data was based on the firing of the (unknown but surely much smaller) number of pyramidal neurons recorded in each experiment. We verified that when estimating the spike rate of 5-15 simulated neurons, the $R^{2}$ values of the simulated rate reconstruction were in the range of those found for experimental LFPs $(0.02-0.05$ for single regressors, $0.05-0.1$ for joint regressors).

The effect of combining the two features is further illustrated in Fig. $6 \mathrm{D}$ by showing a single simulated trace during naturalistic movie simulated stimulation: a linear reconstruction based exclusively on the gamma amplitude would predict the presence of two peaks at the time highlighted by the shaded area (upper row), a reconstruction based exclusively on the delta phase would predict a single symmetric peak (intermediate row), while only combining the two features the real shape of the peak can be reconstructed (lower row). Therefore, consistent with the above considerations on network dynamics, the network model correctly predicts the experimental results that gamma amplitude and delta phase predict the spike rate in a nonredundant way.

Despite the success of linear rate reconstruction, our previous results on network dynamics suggest that a linear model may not be the best way to predict spiking activity. In fact we reported above that the delta phase in naturalistic conditions relates linearly to the rate, while the spike rate increased supra-linearly with the gamma amplitude (or in other words, the gamma amplitude saturated sublinearly at increasing rates, Fig. 3C). We investigated whether the nonlinear relationship between spike rate and gamma amplitude was observed also for real data. Fig. 7A reports the relationship between EEG gamma amplitude and the concurrent spike rate for one representative recording session in V1 of the awake macaque during movie stimulation, and it shows that even a quadratic relationship describes the data much better than a linear fit, in a way that matches the predictions of the neural network model well (Fig. 7C).

The existence of nonlinearities between spike rate and gamma amplitude in both real and simulated data suggests that spike rate prediction may be improved by introducing in the model higher order terms to explain the dependence on gamma amplitude, as follows:

$r=\beta_{\delta} X_{\delta}+\beta_{\gamma}^{(1)} X_{\gamma}+\sum_{n=2}^{N} \beta_{\gamma}^{(n)} X_{\gamma}^{n}+k$

Indeed we found that including a quadratic coefficient $(N=2$ in Eq. (1.8) ) improved the fit relatively to the linear model in 96\% of V1 EEG recording sessions during movie stimulation and $84 \%$ of V1 EEG sessions during spontaneous activity $(F$ test, $p<0.01$ ). The number of non-linear terms that contributed significantly to the reconstruction 
A

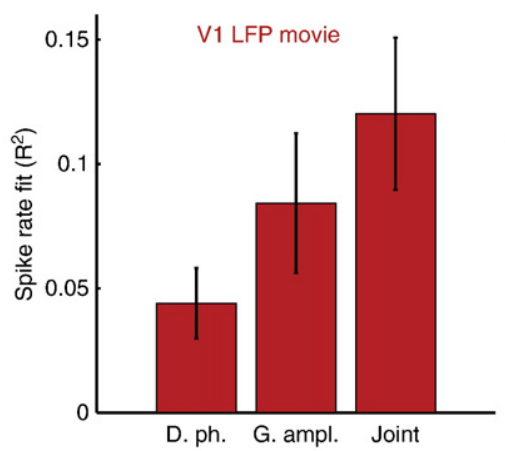

B

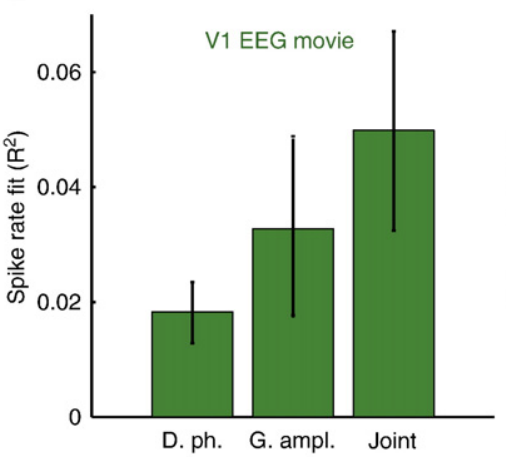

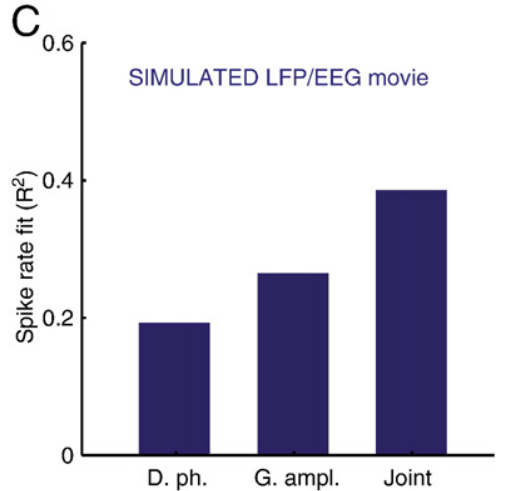

D

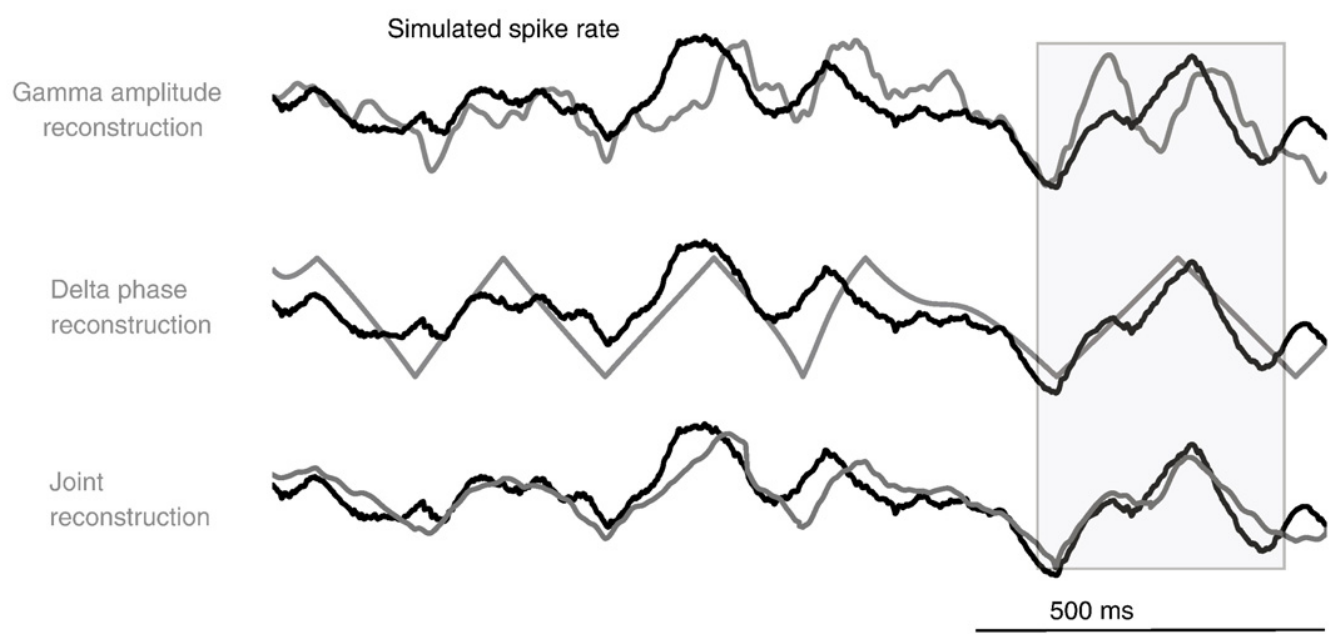

Fig. 6. Spike rate reconstruction from real and simulated EEGs/LFPs signals. (A) $R^{2}$ goodness-of-fit of linear reconstruction of real visual cortical spike rates from the awake monkey during movie stimulation from the concurrently recorded LFPs. The histograms report (as mean \pm SEM over all recording sessions) $R^{2}$ values of linear reconstruction using as regressors LFP delta phase (D. Ph.), gamma amplitude (G. Ampl.) and the two features considered together (Joint) respectively. (B) Same as A, but using concurrently recorded EEGs (rather than LFPs) to estimate visual cortical spike rates. (C) $R^{2}$ goodness-of-fit of linear reconstruction of the output spike rates of the simulated network responding to "naturalistic movie" thalamic inputs. The histograms report (as mean \pm SEM over all simulated data) $R^{2}$ values of linear reconstruction using as regressors simulated LFP/EEG delta phase (D. Ph.), gamma amplitude (G. Pow.) and the two features considered together (Joint) respectively. (D) Evolution of simulated spike rate (in black) and reconstruction of the simulated spike rate from simulated LFP/EEG (in gray) using different regressors: gamma amplitude (top), delta phase ranging between preferred and anti-preferred phase (middle), and both regressors (bottom). Both rate and regressors were convolved with a 100-ms Gaussian window (see text). The shaded box indicates a time region with a spike rate peak whose correct reconstruction requires both regressors.

varied across EEG recordings, and was up to $N=6$ for $50 \%$ of movie sessions and $35 \%$ of spontaneous activity sessions, while simulated data were optimally reconstructed setting $N=4$. In $77 \%$ of movie sessions and $62 \%$ of spontaneous activity sessions, and in simulated data, the non-linear contribution to the best fit coefficient was positive as so the relationship between EEG gamma amplitude and output spike rate was supralinear (Figs. 7A and C). The gain in $R^{2}$ between the non-linear and linear regressor of spike rates based on gamma amplitude during movie stimulation was 33\% and $15 \%$ for real EEGs (Fig. 7B) and for the network model, respectively (Fig. 7D). The gain in $R^{2}$ between the non-linear and linear regressor of spike rates based on gamma amplitude during spontaneous activity was less than the one observed during movie stimulation (15\% and 6\% for real EEGs and for the network model respectively). This is consistent with the predictions of our network model and the mechanisms explained in the previous sections, because the network is less often in the nonlinear spike rate to gamma amplitude conversion regime during spontaneous activity, due to the less sustained input in such a condition (Fig. 3C).

In sum, the network model gives prediction rules of spike rate from EEGs and LFPs which are very consistent with the data, and the investigation of the model dynamics allowed an improvement of the spike rate prediction from real data.
Dependence of cross frequency modulation of spike rates on the stimulus dynamics

According to our model, the finding that phase of the same (delta) slow frequency range is correlated to gamma amplitude and spike rate both during naturalistic movies and spontaneous activity is somehow coincidental, and it happens because during movie stimulation the sensory input fluctuates and entrains the network mostly in the same (slow) frequency regime over which spontaneous and ongoing network fluctuations with the highest power and spatial extent also happen. An important question is how the prediction of spikes from EEGs and LFPs may change with the dynamics of the sensory stimulus. Here, we investigated this issue by using the network model.

We simulated the network responses to a thalamic input with a sinusoidal time course (baseline and amplitude of these input oscillations were set to match the same time-average spike rate and amplitude of peak spectral fluctuation over time of the naturalistic LGN input). In order to understand the impact on the stimulus dynamics on the spike rate control of different EEG-LFP frequencies, we varied the frequency of this input in the range 6-14 Hz. We then examined how the phase of the simulated LFP at different frequencies modulated the output spike rate and gamma amplitude of the simulated network. We found (Fig. 8) that modulation was 


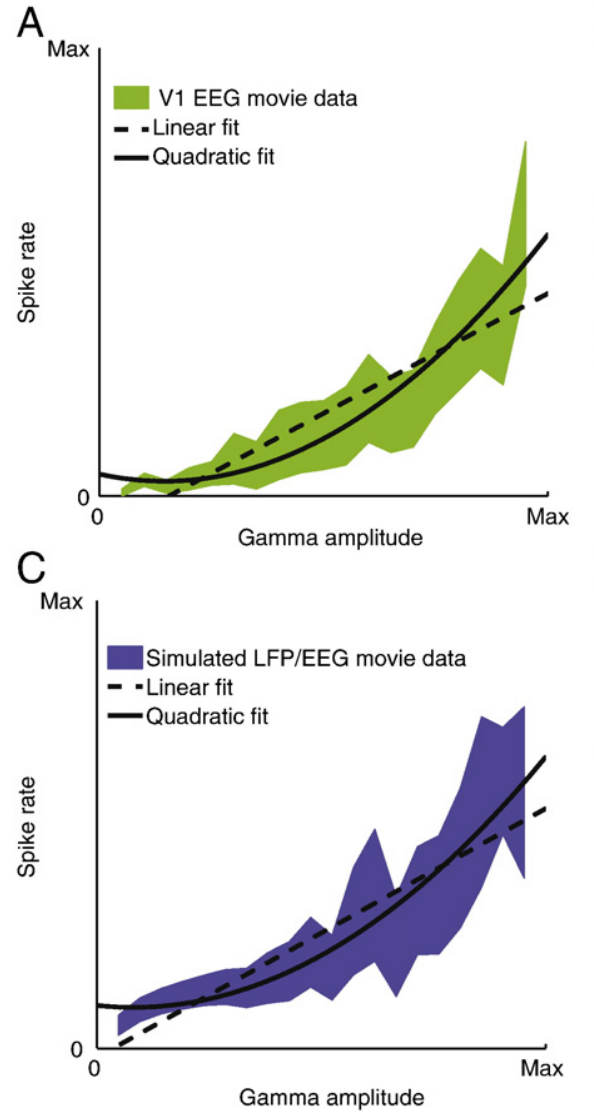

B

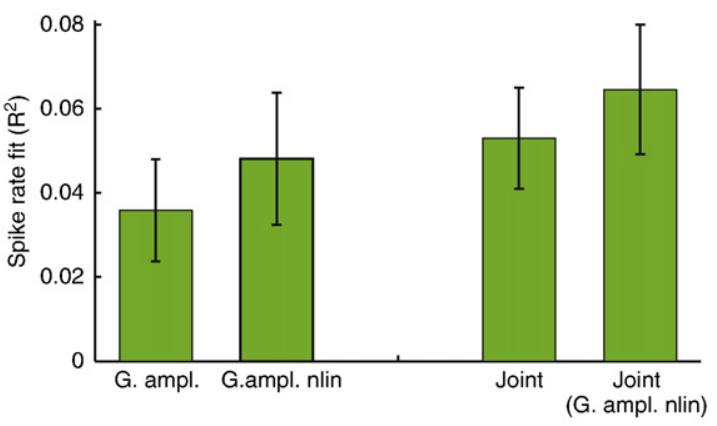

D

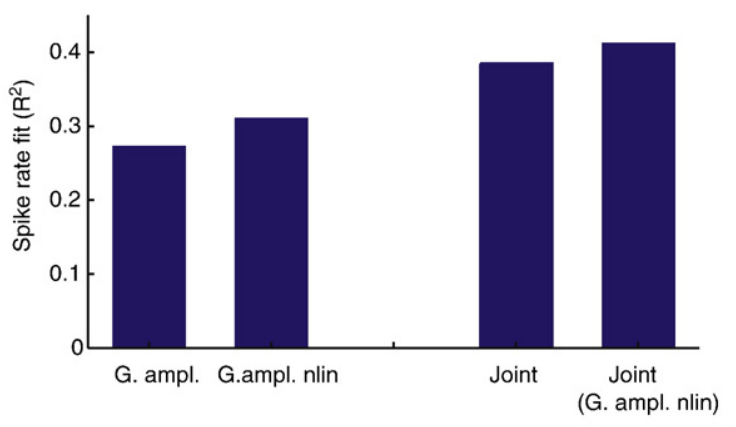

Fig. 7. Non-linear relation between spike rate and gamma amplitude in experiments and simulations. (A) Recorded spike rate as a function of EEG gamma amplitude for a single representative recording session from V1 of the awake monkey during movie stimulation (green area represents mean \pm sd range across all intervals for a given gamma amplitude value). Black lines represent the linear fit (dashed line) and quadratic fit (solid line) of the data. (B) Histograms report (as mean \pm SEM over all recording sessions) $R^{2}$ Goodness-of-fit of the prediction of the time course of primary cortical spike rate during movie stimulation using as regressors different features of the concurrently recorded EEG: linear terms of gamma amplitude (G. Ampl.); the linear and all significant non linear terms of the gamma amplitude (G. Ampl. Nlin.); the linear term of gamma amplitude and the delta phase (Joint); and all significant gamma amplitude terms and the delta phase (Joint (G. Ampl. Nlin.)). The inclusion of the nonlinear terms significantly ( $F$ test, $p<0.01)$ improved the reconstruction of the spike rate from the EEG for $25 / 26$ sessions. (C) Output spike rate of the simulated network in response to a "naturalistic movie" thalamic input as a function of the simulated LFP/EEG gamma amplitude (blue area represents mean \pm sd range across all intervals for a given gamma amplitude value). Black lines represent the linear fit (dashed line) and quadratic fit (solid line) of the data. (D) Histograms report (as mean \pm SEM over all simulated datapoint) $R^{2}$ Goodness-of-fit of the prediction of the time course of the simulated output spike rate in response to movie input using as regressors different features of the simulated EEG/LFP. Notations as in Panel (B).

maximal for LFP phases at the frequency matching that of the stimulus (because of network entrainment to the simulated thalamic input). $R^{2}$ linear regression values obtained using simulated LFP phase at different frequencies were also maximal at the frequency matching that of the stimulus (results not shown). In addition, Fig. 8 shows that in all conditions the phase in the delta range also modulated significantly (though less prominently) the gamma amplitude and the spike rate, because the ongoing unspecific fluctuations that we inserted in the model had maximal power in this low frequency range.

The conclusion of these simulations is that, in addition to the delta phase modulation which should be present whenever ongoing activity is most prominent in this frequency range, the phase at other frequencies may become another prominent correlate of spiking activity in cases in which the stimulus dynamics has the most power outside the delta frequency range. This prediction is easily testable experimentally, and requires a detailed characterization of the stimulus variables which can entrain LFPs and EEGs, and the range of time scales of which this characterization is possible. We however note that any complex naturalistic stimulus will most likely have the most power in the slow frequency range, and thus obtaining a contribution to spike rate predictions from the phase of higher frequencies as that reported in Fig. 8 may not be easy to obtain experimentally.

\section{Discussion}

EEG is one of the most important techniques to record brain activity non-invasively and is a fundamental empirical tool to measure the large-scale dynamics of the human brain during cognitive and sensory functions; yet the exact relationship between the dynamics of the EEG signal and the dynamics of the outputs of local neural computations is still not known. Recently, Whittingstall and Logothetis (2009) investigated this question directly, by recording simultaneously EEGs, LFPs and spikes from visual cortex during naturalistic visual stimulation and in absence of stimuli. They reported that in all cases the combination of EEG or LFP low-frequency phase and high-frequency amplitude yield a significant estimate of real-time spiking activity of cortical neurons. In this article, we developed and reported a novel model explanation of these empirical findings, which led to two main advances: (1) a simple integrate-andfire network model can reproduce these experimental findings obtained in both anaesthetised and awake non-human primate during naturalistic or spontaneous stimulus conditions. (2) The model can be used to better understand the physiological basis of frequency-band coupling, and to therefore better understand the unifying link between EEG, LFP and spiking activity under different experimental conditions. The significance of these studies to investigate brain dynamics is discussed below. 

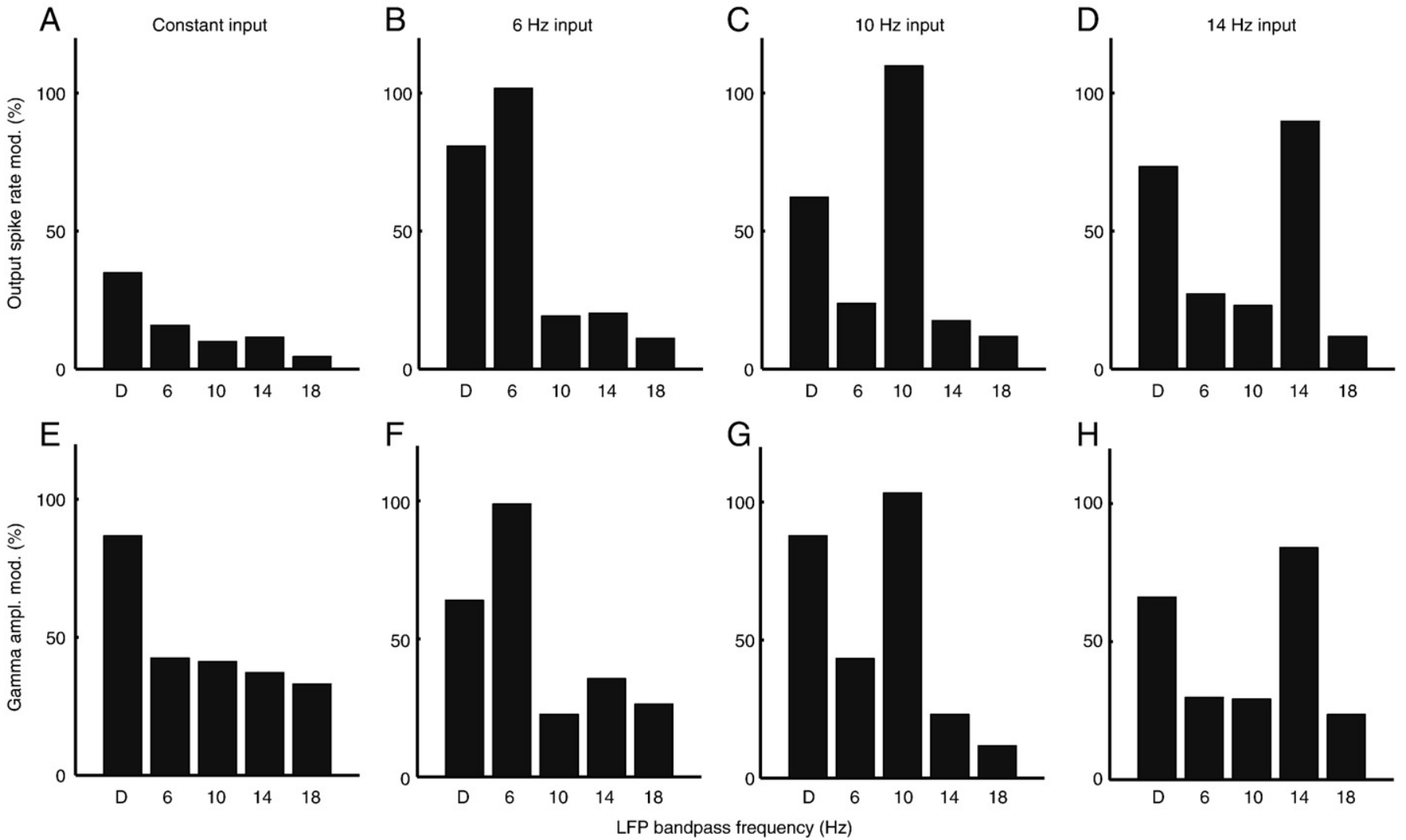

Fig. 8. Modulation of spike rate and gamma amplitude in simulations with high frequency input. (A-D) Simulated spike rate modulation relative to the phase of the delta component $D$ and the phase of LFP/EEG band-passed at $6,10,14$ and $18 \mathrm{~Hz}$, when the network receives cortico-cortical inputs superimposed to a constant input of 1.5 spikes/ms/cell (A), and to sinusoidal inputs of average 1.5 spikes/ms/cell, amplitude 0.8 spikes/ms/cell and frequency $6 \mathrm{~Hz}$ (B), $10 \mathrm{~Hz}$ (C) and $14 \mathrm{~Hz}$ (D). (E, F) Simulated LFP/EEG gamma amplitude modulation relative to the phase of the delta component $D$ and the phase of the simulated LFP/EEG band-passed at $6,10,14$ and $18 \mathrm{~Hz}$, when the network is injected with the same set of stimuli used in (A-D).

\section{Implication for analysis of real EEG, LFP and fMRI recordings}

These results have obvious implications for the interpretation and analysis of EEG experiments, and in particular of how time series of EEG can be used in, for example, deciphering the complex brain activity recorded during a cognitive task. First our model results confirm and corroborate empirical findings and indeed suggest how a reliable estimate of the time course of local spiking activity could be obtained from the single-trial frequency decomposition of EEGs into gamma and delta bands, thereby emphasizing the importance of single-trial frequency analysis in EEGs. Second, our individuation (both in models and in real data) of the nonlinearity in the conversion between spike rate and gamma suggests a practical and very simple way to increase the quality of the estimate of the spike rate by approximately $30 \%$ with a very small computational effort. Third, our hypothesis that a source of modulation of rate by the delta EEG phase arises from fluctuations of the input to a local cortical network due to slow cortical ongoing activity suggests that the phase of the delta band is likely to be a major contributor to spike rate prediction in all experimental conditions. Fourth, our hypothesis that modulation of rate by the EEG phase in slow-frequency bands arise in part from thalamic inputs fluctuations due to entrainment to the stimulus dynamics suggests that in some conditions the phase of EEG/LFP bands other than delta (and whose frequency follows the principal frequencies of stimulus dynamics) may play a significant additional role in spike rate reconstruction to that provided by delta-band phase. This latter point is not only important because it can be helpful to extend the range of validity and quality of model predictions to a number of experimental paradigms beyond naturalistic sensory stimulation and spontaneous activity, but also because it provides very testable predictions of the model which can serve to validate, refute or improve the model assumptions.

A further point of interest is that our model is successful is also predicting spike rate from intracortical LFPs. This point has practical implications because in some clinical applications (such as recording brain activity during human surgeries) it is convenient or possible to record only LFPs and not spiking activity. In such cases, the results provided here give a guideline to obtain from these LFPs simple online estimation of the current level of local spiking activity, which has again very important implications for the clinical evaluation of local functions.

Finally, there is growing evidence (reviewed in He and Raichle, 2009) that slow cortical potentials (similar to the slow oscillations reported here) and their ability to modulate higher frequency oscillations are important neurophysiological correlates of the fMRI BOLD signal. These facts suggest that the model formalism introduced here could potentially be further developed to provide quantitative comparisons to simultaneous recordings of neurophysiological and BOLD signal, and can thus be used to shed further light on how to relate BOLD time series to the output of local neural computations. We plan to pursue this issue in the near future.

\section{Relationship with previous theoretical studies on generation of cross-frequency coupling}

Our model builds on previous theoretical progress on investigating the cross-frequency coupling between the amplitude of faster LFP/ EEG rhythms and the phase of slower rhythms. Since this cross- 
frequency coupling has been first reported in electrophysiological observations in the hippocampus of freely-moving rats, where gamma oscillations occur preferentially at the peak of slower theta oscillations (Bragin et al., 1995; Csicsvari et al., 2003), earlier models of crossfrequency coupling have focused on the hippocampal formation. While gamma oscillations are thought to be generated within hippocampal networks, it is still unclear whether theta oscillations are generated outside or within the hippocampus. White et al. (2000) studied a network model composed of two populations of GABAergic interneurons (expressing GABA receptors with fast and slow kinetics, respectively) that was able to internally generate a mixed thetagamma rhythm. However, cross frequency was much more robust in the presence of the low-frequency components of the input, which modulated generation of gamma rhythms in a way compatible with our proposal. Tsodyks et al. (1997) showed that a Wilson-Cowan type model could generate gamma oscillations that are modulated by a slower theta oscillation which is imposed to the network by an external oscillatory input, because the input drives the network through a Hopf bifurcation, where the network starts to oscillate due to the excitatory-inhibitory loop. Similar cross-frequency coupling phenomena across cortical modules were reported in other theoretical studies of related neural mass models (Jansen and Rit, 1995), and have been used successfully to relate the observed EEG dynamics to the large-scale interactions between brain regions (Chen et al., 2008; David and Friston, 2003; Deco et al., 2008).

Building on this existing knowledge, the work reported here provides several advances. First, we individuated two potential sources of slow modulation of the input and thus excitability to a cortical primary sensory area, namely slow fluctuations in the sensory input from the periphery (possibly related to the stimulus dynamics) and ongoing slow and spatially extended fluctuations of cortical activity. Second, by injecting realistic inputs time series collected from the macaque brain during naturalistic stimulation and during periods of stimulus-free activity into an integrate-and-fire network with sparse recurrent inhibitory and excitatory connections with realistic synaptic time constants, we could demonstrate that our hypotheses about the origin of slow fluctuations of local excitability lead to predictions quantitatively compatible with observed cross-frequency modulation of spiking activity in sensory cortex. Third, and unlike some previous models of generations of oscillations in recurrent networks (Brunel and Wang, 2003; White et al., 2000), we computed and distinguished LFPs/EEGs from input and output spike rates, and this was crucial not only for comparison to experimental data but also to determine the precise mechanisms relating the mesoscopic analog neural signals (such as those from an LFP or an EEG electrode placed near the source of the recorded spiking activity) to the time course of the spike rate, which is the local variable carrying out the information resulting from a neural computation. In particular, we clarified the role of nonlinearities in the conversion from input rate to gamma amplitude and the relationship between delta phase and input strength as potential mechanisms explaining the complementariness in the correlation that slow and fast LFP/EEG oscillations display with spiking activity (Lakatos et al., 2005; Whittingstall and Logothetis, 2009).

A current limitation of our local integrate-and-fire network model is that it may be computationally difficult to extend it in its present form to the study of very large scale interactions between many areas (David and Friston, 2003; Friston, 2001; Sotero et al., 2007), which would allow a study of the role of cross-frequency coupling in organizing large scale cortical dynamics. On the other hand, as demonstrated here this level of modelling permits a very detailed validation against real data of the behaviour of mesoscopic and microscopic signals generated by visual cortex, and is thus a very useful intermediate step for constructing credible larger scale models which can at the same time capture the detailed time course of computation in each area as well as interaction among areas.

\section{Cross-frequency coupling and sensory function}

The coupling of fast gamma oscillations and spike rates with the phase of slower rhythms has been recently reported in visual (Rasch et al., 2008; Whittingstall and Logothetis, 2009) and auditory (Lakatos et al., 2005) cortex, both during spontaneous activity and sensory stimulation. In particular, it has been suggested that this crossfrequency coupling plays a very important role in sensory function and is a crucial aspect of how cortex organizes and groups the different types of information contained at the multiples time scales which are simultaneously present in naturalistic stimuli such as e.g. speech and sound (Kayser et al., 2009; Lakatos et al., 2008; Lakatos et al., 2005). The fact that this phenomenon has been observed in more than one area and both in the presence and absence of sensory stimulation suggests that may reflect a very general feature of cortical dynamics and very basic properties of cortical circuits. Indeed, the mechanisms of generation of cross-frequency coupling that we propose are based on widespread features of cortical circuits and so are likely to be at work in several areas.

An additional view on the role of slow cortical oscillations is that inputs that arrive to a cortical module during the high-excitability delta phase are amplified whereas those arriving during the lowexcitability phase are suppressed, a fact which was suggested to play a role in a number of cognitive functions such as attentional selection (Schroeder and Lakatos, 2009). This view is supported by a recent experiment demonstrating that the pre-stimulus phase of ongoing theta-range EEG oscillation in humans correlates on a trial-by-trial basis with successful visual perception (Busch et al., 2009). We note that the mechanisms of cross-frequency modulation of the output spike rate of a cortical module proposed by our model are in principle compatible with these findings, because in our model a component of the LFP delta oscillation reflects and follows ongoing, stimulus unspecific fluctuations of the cortico-cortical inputs to an area. As a consequence of this model feature, an input which is just subthreshold might become suprathreshold if it arrives at a time when ongoing activity is in an "excitable" state.

\section{Acknowledgments}

We thank M. Maravall for useful comments. This research was supported by the Max Planck Society and by the BMI Project of the Department of Robotics, Brain and Cognitive Sciences at the Italian Institute of Technology.

\section{Appendix A. Supplementary data}

Supplementary data associated with this article can be found, in the online version, at doi:10.1016/j.neuroimage.2009.12.040.

\section{References}

Amit, D.J., Brunel, N., 1997. Model of global spontaneous activity and local structured activity during delay periods in the cerebral cortex. Cereb. Cortex. 7, 237-252.

Belitski, A., Gretton, A., Magri, C., Murayama, Y., Montemurro, M.A., Logothetis, N.K., Panzeri, S., 2008. Low-frequency local field potentials and spikes in primary visual cortex convey independent visual information. J. Neurosci. 28, 5696-5709.

Berens, P., 2009. Circstat: a matlab toolbox for circular statistics. J. Stat. Softw. 31, 1-21.

Bragin, A., Jando, G., Nadasdy, Z., Hetke, J., Wise, K., Buzsaki, G., 1995. Gamma (40-100 Hz) oscillation in the hippocampus of the behaving rat. J. Neurosci. 15, 47-60.

Brunel, N., 2000. Dynamics of sparsely connected networks of excitatory and inhibitory spiking neurons. J. Comput. Neurosci. 8, 183-208.

Brunel, N., Wang, X.J., 2003. What determines the frequency of fast network oscillations with irregular neural discharges? I. Synaptic dynamics and excitation-inhibition balance. J. Neurophysiol. 90, 415-430.

Buhl, E.H., Tamas, G., Szilagyi, T., Stricker, C., Paulsen, O., Somogyi, P., 1997. Effect, number and location of synapses made by single pyramidal cells onto aspiny interneurones of cat visual cortex. J. Physiol. 500 (Pt. 3), 689-713.

Busch, N.A., Dubois, J., VanRullen, R., 2009. The phase of ongoing EEG oscillations predicts visual perception. J. Neurosci. 29, 7869-7876. 
Canolty, R.T., Edwards, E., Dalal, S.S., Soltani, M., Nagarajan, S.S., Kirsch, H.E., Berger M.S., Barbaro, N.M., Knight, R.T., 2006. High gamma power is phase-locked to theta oscillations in human neocortex. Science 313, 1626-1628.

Chen, C.C., Kiebel, S.J., Friston, K.J., 2008. Dynamic causal modelling of induced responses. NeuroImage 41, 1293-1312.

Contreras, D., Steriade, M., 1995. Cellular basis of EEG slow rhythms: a study of dynamic corticothalamic relationships. J. Neurosci. 15, 604-622.

Creutzfeldt, O.D., Watanabe, S., Lux, H.D., 1966a. Relations between EEG phenomena and potentials of single cortical cells. I. Evoked responses after thalamic and erpicortical stimulation. Electroencephalogr. Clin. Neurophysiol. 20, 1-18.

Creutzfeldt, O.D., Watanabe, S., Lux, H.D., 1966b. Relations between EEG phenomena and potentials of single cortical cells. II. Spontaneous and convulsoid activity. Electroencephalogr. Clin. Neurophysiol. 20, 19-37.

Csicsvari, J., Jamieson, B., Wise, K.D., Buzsaki, G., 2003. Mechanisms of gamma oscillations in the hippocampus of the behaving rat. Neuron 37, 311-322.

Dan, Y., Atick, J.J., Reid, R.C., 1996. Efficient coding of natural scenes in the lateral geniculate nucleus: experimental test of a computational theory. J. Neurosci. 16 3351-3362.

David, O., Friston, K.J., 2003. A neural mass model for MEG/EEG: coupling and neuronal dynamics. NeuroImage 20, 1743-1755.

Deco, G., Jirsa, V.K., Robinson, P.A., Breakspear, M., Friston, K., 2008. The dynamic brain from spiking neurons to neural masses and cortical fields. PLoS. Comput. Biol. 4 e1000092.

Dong, D.W., Atick, J.J., 1995. Statistics of natural time-varying images. Netw. Comput. Neural Syst. 6, 345-358.

Friston, K.J., 2001. Brain function, nonlinear coupling, and neuronal transients. Neuroscientist 7, 406-418.

Gabernet, L., Jadhav, S.P., Feldman, D.E., Carandini, M., Scanziani, M., 2005. Somatosensory integration controlled by dynamic thalamocortical feed-forward inhibition. Neuron 48, 315-327.

Geisler, C., Brunel, N., Wang, X.J., 2005. Contributions of intrinsic membrane dynamics to fast network oscillations with irregular neuronal discharges. J. Neurophysiol. 94 4344-4361.

Gil, Z Amitai, Y, 1996. Properties of convergent thalamocortical and intracortical synaptic potentials in single neurons of neocortex. J. Neurosci. 16, 6567-6578.

Goense, J.B., Logothetis, N.K., 2008. Neurophysiology of the BOLD fMRI signal in awake monkeys. Curr. Biol. 18, 631-640.

Granit, R., Kernell, D., Smith, R.S., 1963. Delayed depolarization and the repetitive response to intracellular stimulation of mammalian motoneurones. J. Physiol. 168 890-910.

Gupta, A., Wang, Y., Markram, H., 2000. Organizing principles for a diversity of GABAergic interneurons and synapses in the neocortex. Science 287, 273-278.

He, B.J., Raichle, M.E., 2009. The fMRI signal, slow cortical potential and consciousness. Trends Cogn. Sci. 13, 302-309.

Henrie, J.A., Shapley, R., 2005. LFP power spectra in V1 cortex: the graded effect of stimulus contrast. J. Neurophysiol. 94, 479-490.

Holmgren, C., Harkany, T., Svennenfors, B., Zilberter, Y., 2003. Pyramidal cell communication within local networks in layer 2/3 of rat neocortex. J. Physiol. 551, 139-153.

Jansen, B.H., Rit, V.G., 1995. Electroencephalogram and visual evoked potentia generation in a mathematical model of coupled cortical columns. Biol. Cybern. 73, 357-366.

Jensen, O., Colgin, L.L., 2007. Cross-frequency coupling between neuronal oscillations Trends Cogn. Sci. 11, 267-269.

Juergens, E., Guettler, A., Eckhorn, R., 1999. Visual stimulation elicits locked and induced gamma oscillations in monkey intracortical- and EEG-potentials, but not in human EEG. Exp. Brain Res. 129, 247-259.

Kamondi, A Acsady, L, Wang X. Buzsaki, G, 1998. Theta oscillations in somata and dendrites of hippocampal pyramidal cells in vivo: activity-dependent phaseprecession of action potentials. Hippocampus 8, 244-261.

Katzner, S., Nauhaus, I., Benucci, A., Bonin, V., Ringach, D.L., Carandini, M., 2009. Loca origin of field potentials in visual cortex. Neuron 61, 35-41.

Kayser, C., Montemurro, M.A., Logothetis, N.K., Panzeri, S., 2009. Spike-phase coding boosts and stabilizes information carried by spatial and temporal spike patterns. Neuron 61, 597-608

Klee, M.R., Offenloch, K., Tigges, J., 1965. Cross-correlation analysis of electroencephalographic potentials and slow membrane transients. Science 147, 519-521.

Lakatos, P., Karmos, G., Mehta, A.D., Ulbert, I., Schroeder, C.E., 2008. Entrainment of neuronal oscillations as a mechanism of attentional selection. Science 320, 110-113.
Lakatos, P., Shah, A.S., Knuth, K.H., Ulbert, I., Karmos, G., Schroeder, C.E., 2005. An oscillatory hierarchy controlling neuronal excitability and stimulus processing in the auditory cortex. J. Neurophysiol. 94, 1904-1911.

Latawiec, D., Martin, K.A., Meskenaite, V., 2000. Termination of the geniculocortical projection in the striate cortex of macaque monkey: a quantitative immunoelectron microscopic study. J. Comp. Neurol. 419, 306-319.

Lesica, N.A., Jin, J., Weng, C., Yeh, C.I., Butts, D.A., Stanley, G.B., Alonso, J.M., 2007. Adaptation to stimulus contrast and correlations during natural visual stimulation. Neuron 55, 479-491.

Lisman, J, 2005. The theta/gamma discrete phase code occuring during the hippocampal phase precession may be a more general brain coding scheme Hippocampus 15, 913-922.

Lisman, J.E., Idiart, M.A., 1995. Storage of $7+/-2$ short-term memories in oscillatory subcycles. Science 267, 1512-1515.

Logothetis, N.K., 2003. The underpinnings of the BOLD functional magnetic resonance imaging signal. J. Neurosci. 23, 3963-3971.

Lopes da Silva, F.H., Van Rotterdam, A., 1987. Biophysical aspects of EEG and MEG generation. In: Neidermeyer, E., Lopes da Silva, F. (Eds.), Electroencephalography: Basic Prinicples, clincal applications and related fields. Urban \& Schwarzenberg, Baltimore, pp. 15-28.

Luczak, A., Bartho, P., Harris, K.D., 2009. Spontaneous events outline the realm of possible sensory responses in neocortical populations. Neuron $62,413-425$.

Luczak, A., Bartho, P., Marguet, S.L., Buzsaki, G., Harris, K.D., 2007. Sequential structure of neocortical spontaneous activity in vivo. Proc. Natl. Acad. Sci. U. S. A. 104, 347-352.

Mazzoni, A., Panzeri, S., Logothetis, N.K., Brunel, N., 2008. Encoding of naturalistic stimuli by local field potential spectra in networks of excitatory and inhibitory neurons. PLoS. Comput. Biol. 4, e1000239.

Mitzdorf, U., 1987. Properties of the evoked potential generators: current sourcedensity analysis of visually evoked potentials in the cat cortex. Int. J. Neurosci. 33 33-59.

Montemurro, M.A., Rasch, M.J., Murayama, Y., Logothetis, N.K., Panzeri, S., 2008. Phaseof-firing coding of natural visual stimuli in primary visual cortex. Curr. Biol. 18, 375-380.

Murakami, S., Okada, Y., 2006. Contributions of principal neocortical neurons to magnetoencephalography and electroencephalography signals. J. Physiol. 575 925-936.

Nordlie, E., Gewaltig, M.O., Plesser, H.E., 2009. Towards reproducible descriptions of neuronal network models. PLoS. Comput. Biol. 5, e1000456.

Nunez, P.L., 1981. Electric Fields of the Brain: The Neurophysics of EEG. Oxford Univ. Press, Oxford.

Pettersen, K.H., Einevoll, G.T., 2008. Amplitude variability and extracellular low-pass filtering of neuronal spikes. Biophys. J. 94, 784-802.

Quiroga, R.Q., Nadasdy, Z., Ben-Shaul, Y., 2004. Unsupervised spike detection and sorting with wavelets and superparamagnetic clustering. Neural Comput. 16, 1661-1687.

Rasch, M.J., Gretton, A., Murayama, Y., Maass, W., Logothetis, N.K., 2008. Inferring spike trains from local field potentials. J. Neurophysiol. 99, 1461-1476.

Schroeder, C.E., Lakatos, P., 2009. Low-frequency neuronal oscillations as instruments of sensory selection. Trends Neurosci. 32, 9-18.

Sjostrom, P.J., Turrigiano, G.G., Nelson, S.B., 2001. Rate, timing, and cooperativity jointly determine cortical synaptic plasticity. Neuron 32, 1149-1164.

Sotero, R.C., Trujillo-Barreto, N.J., Iturria-Medina, Y., Carbonell, F., Jimenez, J.C., 2007. Realistically coupled neural mass models can generate EEG rhythms. Neural Comput. 19, 478-512.

Tamas, G., Somogyi, P., Buhl, E.H., 1998. Differentially interconnected networks of GABAergic interneurons in the visual cortex of the cat. J. Neurosci. 18, 4255-4270.

Tsodyks, M.V., Skaggs, W.E., Sejnowski, T.J., McNaughton, B.L., 1997. Paradoxical effects of external modulation of inhibitory interneurons. J. Neurosci. 17, 4382-4388

van Vreeswijk, C., Sompolinsky, H., 1996. Chaos in neuronal networks with balanced excitatory and inhibitory activity. Science $274,1724-1726$.

van Vreeswijk, C., Sompolinsky, H., 1998. Chaotic balanced state in a model of cortical circuits. Neural Comput. 10, 1321-1371.

White, J.A., Banks, M.I., Pearce, R.A., Kopell, N.J., 2000. Networks of interneurons with fast and slow gamma-aminobutyric acid type A (GABAA) kinetics provide substrate for mixed gamma-theta rhythm. Proc. Natl. Acad. Sci. U. S. A. 97, 8128-8133.

Whittingstall, K., Logothetis, N.K., 2009. Frequency-band coupling in surface EEC reflects spiking activity in monkey visual cortex. Neuron 64, 281-289. 